\title{
Linear and integrable nonlinear evolution of the qutrit
}

\author{
Krzysztof Kowalski ${ }^{1}$
}

Received: 17 October 2019 / Accepted: 9 March 2020 / Published online: 18 March 2020

(c) The Author(s) 2020

\section{Abstract}

The nonlinear generalization of the von Neumann equation preserving convexity of the state space is studied in the nontrivial case of the qutrit. This equation can be cast into the integrable classical Riccati system of nonlinear ordinary differential equations. The solutions of such system are investigated in both the linear case corresponding to the standard von Neumann equation and the nonlinear one referring to the generalization of this equation. The analyzed dynamics of the qutrit is rich and includes quasiperiodic motion, multiple equilibria and limit cycles.

Keywords Quantum mechanics · Quantum evolution · Quantum information · Qutrit

\section{Introduction}

In a recent paper [1], the evolution of the density matrix was studied of the form

$$
\rho(t)=\frac{\mathrm{e}^{t(G-i H)} \rho_{0} \mathrm{e}^{t(G+i H)}}{\operatorname{Tr}\left[\mathrm{e}^{t(G-i H)} \rho_{0} \mathrm{e}^{t(G+i H)}\right]},
$$

where $\rho_{0}$ is the $N \times N$ matrix and $H$ and $G$ are Hermitian. The local form of (1.1) is the nonlinear generalization of the von Neumann equation given by

$$
\dot{\rho}(t)=-i[H, \rho(t)]+\{G-\operatorname{Tr}[\rho(t) G], \rho(t)\}, \quad \rho(0)=\rho_{0},
$$

where $\{\cdot, \cdot\}$ designates the anticommutator. We recall [1] that the following nonlinear Schrödinger equation implied by (1.2) when $\rho(t)=|\phi(t)\rangle\langle\phi(t)|$ is the pure state

$$
i \frac{\mathrm{d}}{\mathrm{d} t}|\phi(t)\rangle=(H+i G)|\phi(t)\rangle-i\langle\phi(t)|G| \phi(t)\rangle|\phi(t)\rangle, \quad|\phi(0)\rangle=\left|\phi_{0}\right\rangle,
$$

\section{Krzysztof Kowalski}

kowalski@uni.lodz.pl

1 Department of Theoretical Physics, University of Łódź, ul. Pomorska 149/153, 90-236 Lodz, Poland 
was originally introduced by Gisin [2] as a nonlinear candidate for description of quantum evolution of dissipative systems. It is worthwhile to note that this equation is recognized as "the only sensible candidate for a dissipative Schrödinger equation" [3]. Another application of (1.3) written in the form

$$
i \frac{d}{d t}|\psi(t)\rangle=H|\psi(t)\rangle+\left(1-P_{\psi(t)}\right) U|\psi(t)\rangle,
$$

where $P_{\psi(t)}=|\psi(t)\rangle\langle\psi(t)|$ is the projection operator and $U$ is an arbitrary linear or nonlinear operator, introduced by Grigorenko [4] is the modeling the collapse of the wave function when a measurement is made on a quantum system.

An important property of the complete positive map specified by (1.1) such that

$$
\Phi\left(\rho_{0}\right)=\frac{A(t) \rho_{0} A^{\dagger}(t)}{\operatorname{Tr}\left(A(t) \rho_{0} A^{\dagger}(t)\right)},
$$

where $A(t)=\mathrm{e}^{t(G-i H)}$, is that it preserves the convex structure of the state space. Namely, we have $[1,5,6]$

$$
\Phi\left[\lambda \rho_{1}+(1-\lambda) \rho_{2}\right]=\lambda^{\prime} \Phi\left(\rho_{1}\right)+\left(1-\lambda^{\prime}\right) \Phi\left(\rho_{2}\right),
$$

where $\rho_{1}$ and $\rho_{2}$ are density matrices, and

$$
\lambda^{\prime}=\frac{\operatorname{Tr}\left(A \rho_{1} A^{\dagger}\right)}{\operatorname{Tr}\left\{A\left[\lambda \rho_{1}+(1-\lambda) \rho_{2}\right] A^{\dagger}\right\}} \lambda
$$

satisfies $\lambda^{\prime} \in[0,1]$. Another relevant feature of the dynamics described by Eq. (1.1) is that it maps pure states onto pure ones. We remark that non-unitary evolution in general takes pure states to mixed states. Finally, a desired property of the investigated nonlinear generalization of the von Neumann equation is that it does not allow superluminal messages [7]. We recall that the superluminal signaling is one of the crucial arguments against nonlinear generalizations of quantum mechanics, for example the Schrödinger-Newton equation [8].

We point out that (1.1) is actually the linearization transformation for (1.2). Indeed, the numerator of (1.1) satisfies the linear equation:

$$
\dot{\tilde{\rho}}(t)=-i[H, \tilde{\rho}(t)]+\{G, \tilde{\rho}(t)\}, \quad \rho(0)=\rho_{0},
$$

where $\tilde{\rho}(t)=\mathrm{e}^{t(G-i H)} \rho_{0} \mathrm{e}^{t(G+\mathrm{i} H)}$, so the solution of the nonlinear von Neumann equation (1.2) can be cast into the solution of the linear equation (1.8). Notice that (1.8) with $G$ replaced by $-\frac{1}{2} G$ coincides with the Lamb equation [9] utilized in the theory of the optical maser.

The quantum dynamics described by the nonlinear equation (1.2) was illustrated in [1] by the example of the qubit. To be more specific, we set

$$
\rho_{0}=\frac{1}{2}\left(1+\xi_{0} \cdot \boldsymbol{\sigma}\right)
$$


where $\sigma_{i}, i=1,2,3$ are the Pauli matrices, the dot designates the scalar product, the Bloch vector $\boldsymbol{\xi}_{0} \in \mathbb{R}^{3}$ satisfies $\left|\boldsymbol{\xi}_{0}\right| \leqslant 1$, and

$$
H=\boldsymbol{a} \cdot \boldsymbol{\sigma}, \quad G=\boldsymbol{b} \cdot \boldsymbol{\sigma}
$$

On substituting (1.9) and (1.10) into (1.1), we arrive at the following form of the density matrix $\rho(t)$ :

$$
\rho(t)=\frac{1}{2}(1+\xi(t) \cdot \sigma)
$$

where $\xi(t)$ is an explicit function of $t$ and $\xi_{0}$ (see [1]). On the other hand, inserting (1.11) into the nonlinear von Neumann equation (1.2) we find that $\boldsymbol{\xi}(t)$ is the solution of the following nonlinear system of ordinary differential equations:

$$
\dot{\xi}=2 \boldsymbol{b}+2 \boldsymbol{a} \times \xi-2(\boldsymbol{b} \cdot \xi) \xi, \quad \xi(0)=\xi_{0} .
$$

Thus, it turns out that the nonlinear quantum evolution equation (1.2) can be reduced to the nonlinear classical system (1.12). The system (1.12) with quadratic nonlinearities is called the Riccati system. We remark that as with (1.2) the system (1.12) is quasilinear. The linearization transformation is of the form [1]

$$
\xi(t)=\frac{\eta(t)}{\varphi(t)}
$$

where

$$
\begin{aligned}
& \dot{\varphi}=2 \boldsymbol{b} \cdot \boldsymbol{\eta}, \\
& \dot{\boldsymbol{\eta}}=2 \varphi \boldsymbol{b}+2 \boldsymbol{a} \times \boldsymbol{\eta}, \quad \varphi(0)=1, \quad \boldsymbol{\eta}(0)=\boldsymbol{\xi}_{0} .
\end{aligned}
$$

The solutions to (1.12) were analyzed in a great detail in [1]. In particular, an interesting property of the nonlinear dynamics of the system (1.12) was found - the global asymptotic stability of stationary (equilibrium) solutions corresponding to evolution of the qubit from mixed states to pure ones.

An example of a physical application of the discussed approach is the relativistic quantum spin one-half particle in electromagnetic field analyzed in Ref. [1]. More precisely, the evolution is introduced therein such that the $\rho(t)$ and $A(t)$ in (1.5) are connected with the Bargmann-Michel-Telegdi equations [10] describing relativistic motion of a particle with a magnetic moment in the external electromagnetic field. The vectors $\boldsymbol{a}$ and $\boldsymbol{b}$ from (1.10) are identified with the external magnetic and electric field, respectively. Another interesting physical example is the quantum dynamics with the $s u(1,1)$ Hamiltonian given by (1.10) with time-dependent $\boldsymbol{a}$ and $\boldsymbol{b}$ introduced in Ref. [11], regarded as a Rabi problem with a complex transverse magnetic field. Finally, utilizing a nonlinear generalization of the Gorini-Kossakowski-Sudarshan-Lindblad equation in the case of the qubit, based on the nonlinear von Neumann equation (1.2) as a point of departure, the extension of the celebrated Jaynes-Cummings model was 
introduced in Ref. [12] describing the interaction of a two-level atom with a single mode of the electromagnetic field.

In this work following the approach taken up in the case of the qubit, we study the much more complicated case of the qutrit. The paper is organized as follows. In Sect. 2, we introduce the nonlinear system that is the counterpart of (1.12) in the case with the qutrit. Section 3 is devoted to the linear case referring to the standard von Neumann equation. Section 4 deals with the nonlinear system. All the necessary identities corresponding to the $s u(3)$ algebra are collected in "Appendix".

\section{Nonlinear evolution of a qutrit}

Consider now the special case of a qutrit. The well-known generalized Bloch sphere representation for a qutrit is specified by the density matrix:

$$
\rho=\frac{1}{3}(1+\sqrt{3} \xi \cdot \lambda)
$$

where $\lambda_{i}, i=1,2, \ldots, 8$, are the Gell-Mann matrices (see "Appendix"), and the state space $\Omega$ is given by [13]

$$
\Omega=\left\{\xi \in \mathbb{R}^{8}: 3 \xi^{2}-2 \xi \cdot(\xi * \xi) \leqslant 1, \quad \xi^{2} \leqslant 1\right\},
$$

where $\boldsymbol{a} * \boldsymbol{b}$ is the symmetric star product of vectors $\boldsymbol{a}, \boldsymbol{b} \in \mathbb{R}^{8}$ described in "Appendix." In opposition to the qubit states, the boundary $\partial \Omega$ of the space of states $\Omega$ specified by

$$
\partial \Omega=\left\{\xi \in \mathbb{R}^{8}: 3 \xi^{2}-2 \xi \cdot(\xi * \xi)=1, \quad \xi^{2} \leqslant 1\right\}
$$

contains both the pure states characterized by

$$
\partial \Omega_{p}=\left\{\xi \in \mathbb{R}^{8}: \xi^{2}=1, \quad \xi * \xi=\xi\right\},
$$

and mixed states:

$$
\partial \Omega_{m}=\left\{\boldsymbol{\xi} \in \mathbb{R}^{8}: 3 \xi^{2}-2 \xi \cdot(\xi * \xi)=1, \quad \xi^{2}<1\right\},
$$

The state space $\Omega$ is five-dimensional, and its boundary $\partial \Omega$ is four-dimensional. Taking into account that $\partial \Omega_{p}$ is the coset space $S U(3) / U(2)$, one can find the following useful parametrization of $\partial \Omega_{p}$ by angles $\alpha, \beta, \gamma$ and $\delta$ :

$$
\begin{aligned}
\xi=\sqrt{3} & (\sin \alpha \sin \beta \cos \alpha \cos (\delta-\gamma), \sin \alpha \sin \beta \cos \alpha \sin (\delta-\gamma), \\
& \frac{1}{2}\left(\cos ^{2} \alpha \sin ^{2} \beta-\sin ^{2} \alpha\right), \cos ^{2} \alpha \cos \beta \sin \beta \cos \gamma, \\
& -\cos ^{2} \alpha \cos \beta \sin \beta \sin \gamma, \cos \beta \cos \alpha \sin \alpha \cos \delta, \\
& \left.-\cos \beta \cos \alpha \sin \alpha \sin \delta, \frac{1}{2 \sqrt{3}}\left(\cos ^{2} \alpha \sin ^{2} \beta+\sin ^{2} \alpha-2 \cos ^{2} \alpha \cos ^{2} \beta\right)\right) .
\end{aligned}
$$


We remark that there exist in the literature the alternative parametrizations of $\partial \Omega_{p}$ (see for example [14]).

Now proceeding analogously as with the qubit states, we set

$$
\rho_{0}=\frac{1}{3}\left(1+\sqrt{3} \xi_{0} \cdot \lambda\right), \quad \rho(t)=\frac{1}{3}(1+\sqrt{3} \xi(t) \cdot \lambda),
$$

and

$$
H=\boldsymbol{a} \cdot \boldsymbol{\lambda}, \quad G=\boldsymbol{b} \cdot \boldsymbol{\lambda} .
$$

On substituting (2.7) and (2.8) into (1.2) and using the identities (A.30), (A.32) and (A.33), we arrive at the following Riccati system of nonlinear ordinary differential equations:

$$
\dot{\xi}=\frac{2}{\sqrt{3}} \boldsymbol{b}+\frac{2}{\sqrt{3}} \boldsymbol{a} \wedge \boldsymbol{\xi}+\frac{2}{\sqrt{3}} \boldsymbol{b} * \boldsymbol{\xi}-\frac{4}{\sqrt{3}}(\boldsymbol{b} \cdot \boldsymbol{\xi}) \boldsymbol{\xi}, \quad \boldsymbol{\xi}(0)=\boldsymbol{\xi}_{0},
$$

where $\boldsymbol{a} \wedge \boldsymbol{b}$ is the antisymmetric product of vectors $\boldsymbol{a}$ and $\boldsymbol{b}$ (see "Appendix"). It thus appears that the nonlinear quantum evolution equation (1.2) with $\rho(t), H$ and $G$ given by (2.7) and (2.8), respectively, reduces to the nonlinear classical system (2.9).

\section{Linear evolution of a qutrit: periodic and quasiperiodic solutions}

We now restrict to the case $\boldsymbol{b}=0$. The system (2.9) reduces then to the linear one corresponding to the linear von Neumann equation. Namely, we have

$$
\dot{\xi}=\frac{2}{\sqrt{3}} a \wedge \xi, \quad \xi(0)=\xi_{0} .
$$

In opposition to the evolution of the qubit discussed in [1], even in such a linear case the problem of finding the solution to (3.1) by means of the transformation (1.1) is in general complex and leads to cumbersome formulas. To be more specific, consider first the problem of calculating the exponential $\mathrm{e}^{\boldsymbol{a} \cdot \boldsymbol{\lambda}}$. Referring to (1.1) and (2.1), we point out that in view of (A.19) and (A.24) an arbitrary power of $\boldsymbol{a} \cdot \boldsymbol{\lambda}$ has the expansion of the form

$$
(\boldsymbol{a} \cdot \boldsymbol{\lambda})^{n}=c_{n}+d_{n} \boldsymbol{a} \cdot \boldsymbol{\lambda}+e_{n}(\boldsymbol{a} * \boldsymbol{a}) \cdot \boldsymbol{\lambda}, \quad n=0,1,2, \ldots,
$$

where $c_{n}, d_{n}$ and $e_{n}$ are the scalar coefficients. On using (A.19), (A.24) and (A.30), we arrive at the following system of recurrence equation:

$$
\begin{aligned}
c_{n+1} & =\frac{2}{3} \boldsymbol{a}^{2} d_{n}+\frac{2}{3} \boldsymbol{a} \cdot(\boldsymbol{a} * \boldsymbol{a}) e_{n}, \\
d_{n+1} & =\frac{1}{\sqrt{3}} \boldsymbol{a}^{2} e_{n}+c_{n}, \\
e_{n+1} & =\frac{1}{\sqrt{3}} d_{n},
\end{aligned}
$$


subject to the initial data $c_{0}=1, c_{1}=0, d_{0}=0, d_{1}=1, e_{0}=0, e_{1}=0$. From (3.3), we get the recursive relation:

$$
d_{n+3}-\boldsymbol{a}^{2} d_{n+1}-\frac{2}{3 \sqrt{3}} \boldsymbol{a} \cdot(\boldsymbol{a} * \boldsymbol{a}) d_{n}=0 .
$$

The characteristic equation corresponding to (3.4) is of the form

$$
x^{3}-a^{2} x-\frac{2}{3 \sqrt{3}} \boldsymbol{a} \cdot(\boldsymbol{a} * \boldsymbol{a})=0 .
$$

The discriminant of the cubic equation (3.5) is

$$
Q=\frac{1}{27}\left\{[\boldsymbol{a} \cdot(\boldsymbol{a} * \boldsymbol{a})]^{2}-|\boldsymbol{a}|^{6}\right\}
$$

where $|\boldsymbol{a}|$ designates the norm of the vector $\boldsymbol{a}$. The discriminant (3.6) fulfills $Q \leqslant 0$. The simplest case $Q=0$ refers to the condition $\boldsymbol{a} * \boldsymbol{a}= \pm|\boldsymbol{a}| \boldsymbol{a}$ and will be discussed later. For $Q<0$, we have the trigonometric solution [15] to (3.5) such that

$$
\begin{aligned}
x_{1} & =\frac{2}{\sqrt{3}}|\boldsymbol{a}| \cos \frac{\alpha}{3}, \\
x_{2,3} & =-\frac{2}{\sqrt{3}}|\boldsymbol{a}| \cos \left(\frac{\alpha}{3} \pm \frac{\pi}{3}\right),
\end{aligned}
$$

where

$$
\cos \alpha=\frac{\boldsymbol{a} \cdot(\boldsymbol{a} * \boldsymbol{a})}{|\boldsymbol{a}|^{3}} .
$$

Using (3.3), (3.4) and (3.7), we find after some calculation

$$
\begin{aligned}
c_{n}= & \frac{1}{12} \frac{4 \cos ^{2} \frac{\alpha}{3}-1}{\cos \left(\frac{\alpha}{3}+\frac{\pi}{6}\right) \cos \left(\frac{\alpha}{3}-\frac{\pi}{6}\right)}\left(\frac{2}{\sqrt{3}}|\boldsymbol{a}| \cos \frac{\alpha}{3}\right)^{n} \\
& +\frac{1}{12} \frac{-4 \sin ^{2}\left(\frac{\alpha}{3}-\frac{\pi}{6}\right)+1}{\cos \left(\frac{\alpha}{3}+\frac{\pi}{6}\right) \sin \frac{\alpha}{3}}\left[-\frac{2}{\sqrt{3}}|\boldsymbol{a}| \cos \left(\frac{\alpha}{3}+\frac{\pi}{3}\right)\right]^{n} \\
& +\frac{1}{12} \frac{4 \sin ^{2}\left(\frac{\alpha}{3}+\frac{\pi}{6}\right)-1}{\cos \left(\frac{\alpha}{3}-\frac{\pi}{6}\right) \sin \frac{\alpha}{3}}\left[-\frac{2}{\sqrt{3}}|\boldsymbol{a}| \cos \left(\frac{\alpha}{3}-\frac{\pi}{3}\right)\right]^{n}, \\
d_{n}= & \frac{1}{2 \sqrt{3}} \frac{\cos \frac{\alpha}{3}}{|\boldsymbol{a}| \cos \left(\frac{\alpha}{3}+\frac{\pi}{6}\right) \cos \left(\frac{\alpha}{3}-\frac{\pi}{6}\right)}\left(\frac{2}{\sqrt{3}}|\boldsymbol{a}| \cos \frac{\alpha}{3}\right)^{n} \\
& -\frac{1}{2 \sqrt{3}} \frac{\sin \left(\frac{\alpha}{3}-\frac{\pi}{6}\right)}{|\boldsymbol{a}| \cos \left(\frac{\alpha}{3}+\frac{\pi}{6}\right) \sin \frac{\alpha}{3}}\left[-\frac{2}{\sqrt{3}}|\boldsymbol{a}| \cos \left(\frac{\alpha}{3}+\frac{\pi}{3}\right)\right]^{n} \\
& -\frac{1}{2 \sqrt{3}} \frac{\sin \left(\frac{\alpha}{3}+\frac{\pi}{6}\right)}{|\boldsymbol{a}| \cos \left(\frac{\alpha}{3}-\frac{\pi}{6}\right) \sin \frac{\alpha}{3}}\left[-\frac{2}{\sqrt{3}}|\boldsymbol{a}| \cos \left(\frac{\alpha}{3}-\frac{\pi}{3}\right)\right]^{n},
\end{aligned}
$$




$$
\begin{aligned}
e_{n}= & \frac{1}{4 \sqrt{3}} \frac{1}{\boldsymbol{a}^{2} \cos \left(\frac{\alpha}{3}+\frac{\pi}{6}\right) \cos \left(\frac{\alpha}{3}-\frac{\pi}{6}\right)}\left(\frac{2}{\sqrt{3}}|\boldsymbol{a}| \cos \frac{\alpha}{3}\right)^{n} \\
& -\frac{1}{4 \sqrt{3}} \frac{1}{\boldsymbol{a}^{2} \cos \left(\frac{\alpha}{3}+\frac{\pi}{6}\right) \sin \frac{\alpha}{3}}\left[-\frac{2}{\sqrt{3}}|\boldsymbol{a}| \cos \left(\frac{\alpha}{3}+\frac{\pi}{3}\right)\right]^{n} \\
& +\frac{1}{4 \sqrt{3}} \frac{1}{\boldsymbol{a}^{2} \cos \left(\frac{\alpha}{3}-\frac{\pi}{6}\right) \sin \frac{\alpha}{3}}\left[-\frac{2}{\sqrt{3}}|\boldsymbol{a}| \cos \left(\frac{\alpha}{3}-\frac{\pi}{3}\right)\right]^{n} .
\end{aligned}
$$

An immediate consequence of (3.9), (3.10) and (3.11) is the following formula for the exponential $\mathrm{e}^{\tau \boldsymbol{a} \cdot \boldsymbol{\lambda}}$ :

$$
\begin{aligned}
\mathrm{e}^{\tau \boldsymbol{a} \cdot \boldsymbol{\lambda}}= & \frac{1}{12} \frac{4 \cos ^{2} \frac{\alpha}{3}-1}{\cos \left(\frac{\alpha}{3}+\frac{\pi}{6}\right) \cos \left(\frac{\alpha}{3}-\frac{\pi}{6}\right)} \exp \left(\frac{2 \tau}{\sqrt{3}}|\boldsymbol{a}| \cos \frac{\alpha}{3}\right) \\
& +\frac{1}{12} \frac{-4 \sin ^{2}\left(\frac{\alpha}{3}-\frac{\pi}{6}\right)+1}{\cos \left(\frac{\alpha}{3}+\frac{\pi}{6}\right) \sin \frac{\alpha}{3}} \exp \left[-\frac{2 \tau}{\sqrt{3}}|\boldsymbol{a}| \cos \left(\frac{\alpha}{3}+\frac{\pi}{3}\right)\right] \\
& +\frac{1}{12} \frac{4 \sin ^{2}\left(\frac{\alpha}{3}+\frac{\pi}{6}\right)-1}{\cos \left(\frac{\alpha}{3}-\frac{\pi}{6}\right) \sin \frac{\alpha}{3}} \exp \left[-\frac{2 \tau}{\sqrt{3}}|\boldsymbol{a}| \cos \left(\frac{\alpha}{3}-\frac{\pi}{3}\right)\right] \\
& +\left(\frac{1}{2 \sqrt{3}} \frac{\cos \frac{\alpha}{3}}{|\boldsymbol{a}| \cos \left(\frac{\alpha}{3}+\frac{\pi}{6}\right) \cos \left(\frac{\alpha}{3}-\frac{\pi}{6}\right)} \exp \left(\frac{2 \tau}{\sqrt{3}}|\boldsymbol{a}| \cos \frac{\alpha}{3}\right)\right. \\
& -\frac{1}{2 \sqrt{3}} \frac{\sin \left(\frac{\alpha}{3}-\frac{\pi}{6}\right)}{|\boldsymbol{a}| \cos \left(\frac{\alpha}{3}+\frac{\pi}{6}\right) \sin \frac{\alpha}{3}} \exp \left[-\frac{2 \tau}{\sqrt{3}}|\boldsymbol{a}| \cos \left(\frac{\alpha}{3}+\frac{\pi}{3}\right)\right] \\
& \left.-\frac{1}{2 \sqrt{3}} \frac{\sin \left(\frac{\alpha}{3}+\frac{\pi}{6}\right)}{|\boldsymbol{a}| \cos \left(\frac{\alpha}{3}-\frac{\pi}{6}\right) \sin \frac{\alpha}{3}} \exp \left[-\frac{2 \tau}{\sqrt{3}}|\boldsymbol{a}| \cos \left(\frac{\alpha}{3}-\frac{\pi}{3}\right)\right]\right) \boldsymbol{a} \cdot \boldsymbol{\lambda} \\
& +\left(\frac{1}{4 \sqrt{3}} \frac{1}{\boldsymbol{a}^{2} \cos \left(\frac{\alpha}{3}+\frac{\pi}{6}\right) \cos \left(\frac{\alpha}{3}-\frac{\pi}{6}\right)} \exp \left(\frac{2}{\sqrt{3}}|\boldsymbol{a}| \cos \frac{\alpha}{3}\right)\right. \\
& -\frac{1}{4 \sqrt{3}} \frac{1}{\boldsymbol{a}^{2} \cos \left(\frac{\alpha}{3}+\frac{\pi}{6}\right) \sin \frac{\alpha}{3}} \exp \left[-\frac{2 \tau}{\sqrt{3}}|\boldsymbol{a}| \cos \left(\frac{\alpha}{3}+\frac{\pi}{3}\right)\right] \\
& \left.+\frac{1}{4 \sqrt{3}} \frac{1}{\boldsymbol{a}^{2} \cos \left(\frac{\alpha}{3}-\frac{\pi}{6}\right) \sin \frac{\alpha}{3}} \exp \left[-\frac{2 \tau}{\sqrt{3}}|\boldsymbol{a}| \cos \left(\frac{\alpha}{3}-\frac{\pi}{3}\right)\right]\right)(\boldsymbol{a} * \boldsymbol{a}) \cdot \boldsymbol{\lambda} . \\
& (3 .)
\end{aligned}
$$

As a matter of fact, setting $\tau=-i t$ in (3.12) and making use of (1.1), (2.7) and (2.8) one can obtain the solution to (3.1). Nevertheless, as mentioned earlier the formulas are cumbersome and we decided not to present them herein. The numerical integration of (3.1) shows that the typical trajectory is the quasiperiodic one such as that depicted in Fig. 1. We remark that quasiperiodic trajectories were absent in the case with the evolution of the qubit [1]. The remaining cases include periodic (see Fig. 2) and stationary solutions. In the next sections, we introduce explicit solutions to (3.1) in the special cases with $\boldsymbol{a} * \boldsymbol{a}= \pm|\boldsymbol{a}| \boldsymbol{a}, \boldsymbol{a} \cdot(\boldsymbol{a} * \boldsymbol{a})=0$, and the diagonal generator $\boldsymbol{a} \cdot \boldsymbol{\lambda}$. 

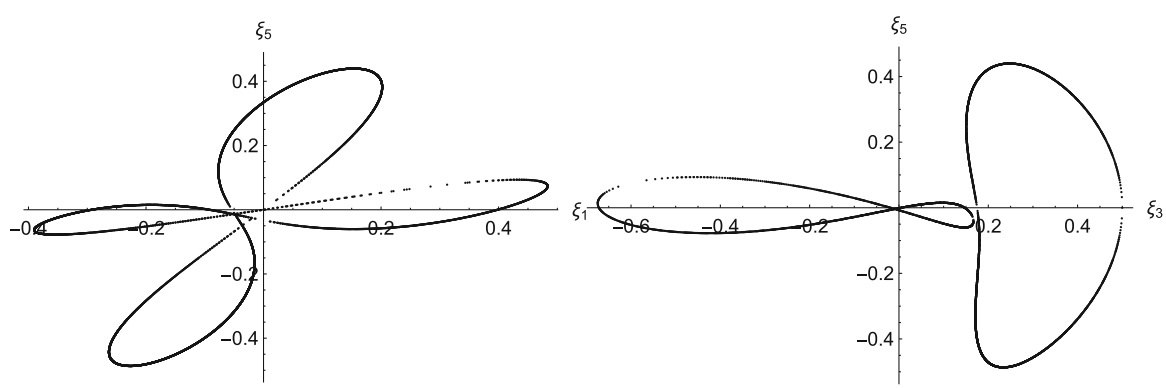

Fig. 1 Quasiperiodic trajectory of the system (3.1) with $\boldsymbol{a}=(0,1,0,-1,0.3,0,1,0)$ and $\boldsymbol{\xi}_{0}=$ $\left(0,0, \frac{1}{2}, 0,0,0,0, \frac{1}{2}\right)$. Left: the projection of the Poincaré section of the trajectory onto the $\left(\xi_{1}, \xi_{5}\right)$ plane. Right: the projection of the Poincaré section onto the $\left(\xi_{3}, \xi_{5}\right)$ plane. The Poincaré section is defined by the hyperplane $\boldsymbol{n} \cdot\left(\boldsymbol{\xi}-\xi^{(0)}\right)=0$, where the normal vector $\boldsymbol{n}=(0,1,0,0,0,0,0,0)$ and $\xi^{(0)}=(0,0,0,0,0,0,0,0)$

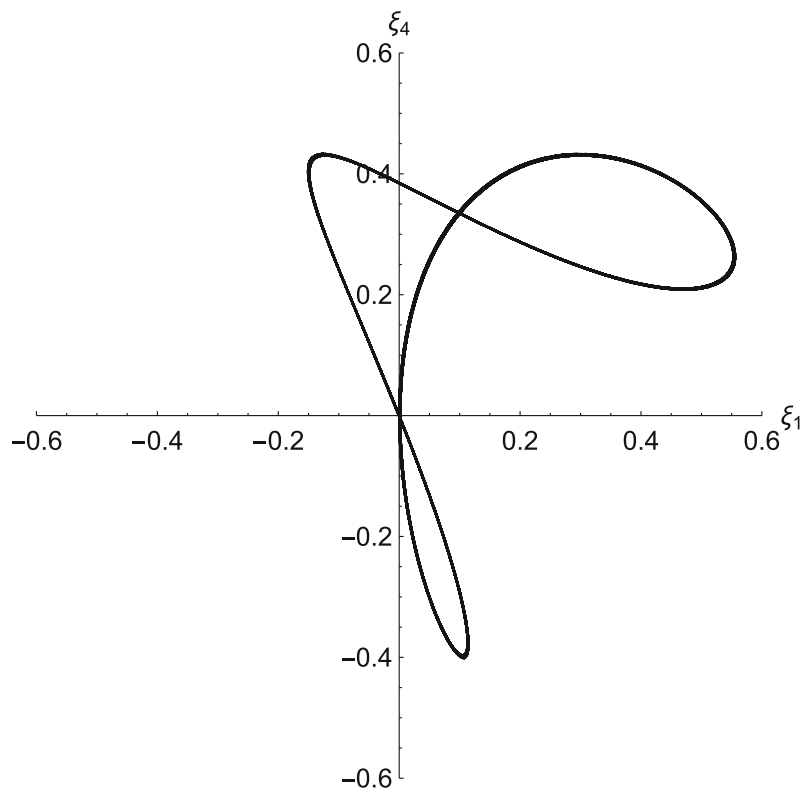

Fig. 2 The periodic solution of the system (3.1) corresponding to $\boldsymbol{a} \cdot(\boldsymbol{a} * \boldsymbol{a})=0$, given by (3.28), where $\boldsymbol{a}=(1,0,1,1,-1,1,1,0)$ and $\xi_{0}=\left(0,0, \frac{\sqrt{3}}{2}, 0,0,0,0, \frac{1}{2}\right)$. The projection of the trajectory onto the $\left(\xi_{1}, \xi_{4}\right)$ plane

We now return to (3.1). Using (A.26), we find $\xi^{2}=$ const. Further, taking into account (A.18), (A.26) and (A.27) we can demonstrate (see also [16]) that $\boldsymbol{\xi} \cdot(\boldsymbol{\xi} * \boldsymbol{\xi})$ is the first integral of (3.1) as well. In view of (2.2), (2.4) and (2.5), we conclude that under the unitary linear evolution given by the generator $\boldsymbol{a} \cdot \boldsymbol{\lambda}$, the pure states remain pure ones - this was shown in the case of the general nonlinear evolution described globally by Eq. (1.1). Furthermore, it turns out that mixed states are also stable under the linear unitary evolution including the case of the mixed states on the boundary (2.5) $\partial \Omega_{m}$. 
Consider now the stationary solutions to (3.1). By virtue of (A.20), the most general form of these solutions is

$$
\bar{\xi}=\mu \boldsymbol{a}+v \boldsymbol{a} * \boldsymbol{a},
$$

where $\mu$ and $v$ are constants. From (2.2), it follows that the states $\bar{\xi}$ correspond to the following region in the $(\mu, \nu)$ plane:

$$
\begin{aligned}
& \boldsymbol{a}^{2} \mu^{2}+|\boldsymbol{a}|^{4} v^{2}+2 \boldsymbol{a} \cdot(\boldsymbol{a} * \boldsymbol{a}) \mu v \leqslant 1, \\
& -2 \boldsymbol{a} \cdot(\boldsymbol{a} * \boldsymbol{a}) \mu^{3}+2\left\{|\boldsymbol{a}|^{6}-2[\boldsymbol{a} \cdot(\boldsymbol{a} * \boldsymbol{a})]^{2}\right\} v^{3}-6|\boldsymbol{a}|^{4} \mu^{2} v+3 \boldsymbol{a}^{2} \mu^{2}+3|\boldsymbol{a}|^{4} v^{2} \\
& \quad+6 \boldsymbol{a} \cdot(\boldsymbol{a} * \boldsymbol{a})\left(1-\boldsymbol{a}^{2}\right) \mu v \leqslant 1 .
\end{aligned}
$$

The pure states (2.4) lying in the boundary $\partial \Omega_{p}$ are given by (3.13), where $\mu$ and $v$ are the solution of the system:

$$
\begin{aligned}
& \boldsymbol{a}^{2} \mu^{2}+|\boldsymbol{a}|^{4} v^{2}+2 \boldsymbol{a} \cdot(\boldsymbol{a} * \boldsymbol{a}) \mu \nu=1, \\
& \boldsymbol{a} \cdot(\boldsymbol{a} * \boldsymbol{a}) \mu^{3}+\left\{2[\boldsymbol{a} \cdot(\boldsymbol{a} * \boldsymbol{a})]^{2}-|\boldsymbol{a}|^{6}\right\} v^{3}+3|\boldsymbol{a}|^{4} \mu^{2} v+3 \boldsymbol{a}^{2} \boldsymbol{a} \cdot(\boldsymbol{a} * \boldsymbol{a}) \mu \nu=1,
\end{aligned}
$$

where the second equation of (3.15) is a consequence of the relation $\bar{\xi} \cdot(\bar{\xi} * \bar{\xi})=1$. Finally, the mixed states $(2.5)$ on the boundary $\partial \Omega_{m}$ are specified by

$$
\begin{aligned}
& \boldsymbol{a}^{2} \mu^{2}+|\boldsymbol{a}|^{4} v^{2}+2 \boldsymbol{a} \cdot(\boldsymbol{a} * \boldsymbol{a}) \mu v<1, \\
& -2 \boldsymbol{a} \cdot(\boldsymbol{a} * \boldsymbol{a}) \mu^{3}+2\left\{|\boldsymbol{a}|^{6}-2[\boldsymbol{a} \cdot(\boldsymbol{a} * \boldsymbol{a})]^{2}\right\} v^{3}-6|\boldsymbol{a}|^{4} \mu^{2} v+3 \boldsymbol{a}^{2} \mu^{2}+3|\boldsymbol{a}|^{4} v^{2} \\
& \quad+6 \boldsymbol{a} \cdot(\boldsymbol{a} * \boldsymbol{a})\left(1-\boldsymbol{a}^{2}\right) \mu v=1 .
\end{aligned}
$$

The concrete examples of the stationary solutions to the system (3.1) are discussed in the following sections.

\subsection{The case of $a * a=|a| a$}

We first recall that the condition $\boldsymbol{a} * \boldsymbol{a}=|\boldsymbol{a}| \boldsymbol{a}$ refers to $Q=0$ in (3.6). Taking into account (3.2), we find that in this case

$$
(\boldsymbol{a} \cdot \boldsymbol{\lambda})^{n}=c_{n}+d_{n} \boldsymbol{a} \cdot \boldsymbol{\lambda}, \quad n=0,1,2, \ldots
$$

Hence, proceeding as with (3.2) and using (A.31) we obtain the system of recurrence equations satisfied by $c_{n}$ and $d_{n}$. Solving the second-order recurrence satisfied by $d_{n}$, we get

$$
\mathrm{e}^{\tau \boldsymbol{a} \cdot \boldsymbol{\lambda}}=\frac{1}{3} \mathrm{e}^{\frac{2 \sqrt{3}}{3} \tau|\boldsymbol{a}|}+\frac{2}{3} \mathrm{e}^{-\frac{\sqrt{3}}{3} \tau|\boldsymbol{a}|}+\frac{1}{\sqrt{3}|\boldsymbol{a}|}\left(\mathrm{e}^{\frac{2 \sqrt{3}}{3} \tau|\boldsymbol{a}|}-\mathrm{e}^{-\frac{\sqrt{3}}{3} \tau|\boldsymbol{a}|}\right) \boldsymbol{a} \cdot \boldsymbol{\lambda}
$$


On putting $\tau=-i t$ and making use of (1.1), (2.7) and (2.8), we arrive after some calculation to the following formula for the solution to (3.1):

$$
\begin{aligned}
\boldsymbol{\xi}(t)= & \left(\frac{1}{3}+\frac{2}{3} \cos (t \sqrt{3}|\boldsymbol{a}|)\right) \boldsymbol{\xi}_{0}+\frac{2}{3|\boldsymbol{a}|} \sin (t \sqrt{3}|\boldsymbol{a}|) \boldsymbol{a} \wedge \boldsymbol{\xi}_{0} \\
& +(1-\cos (t \sqrt{3}|\boldsymbol{a}|))\left(-\frac{2}{3|\boldsymbol{a}|} \boldsymbol{a} * \boldsymbol{\xi}_{0}+\frac{4}{3 \boldsymbol{a}^{2}}\left(\boldsymbol{a} \cdot \boldsymbol{\xi}_{0}\right) \boldsymbol{a}\right)
\end{aligned}
$$

Using (3.13) and (3.15), we find that the system (3.1) has in the discussed case the stationary solution representing the pure state of the form:

$$
\bar{\xi}=\frac{a}{|a|} .
$$

Evidently, the stationary solution (3.20) is not asymptotically stable. (This is not any limit of $t$ going to infinity of (3.19).) Furthermore, the stationary solution representing the mixed state on the boundary of the state space is given by

$$
\bar{\xi}^{\prime}=-\frac{a}{2|a|}
$$

Finally, we have a family of stationary solutions corresponding to mixed states in the interior of the space of states such that

$$
\bar{\xi}^{\prime \prime}=-\frac{a}{\sigma|a|},
$$

where $\sigma>2$. Of course, $\overline{\boldsymbol{\xi}}^{\prime \prime}$ approaches 0 referring to maximally mixed state in the limit $\sigma \rightarrow \infty$.

The projections of the periodic trajectory (3.19) onto the planes $\left(\xi_{i}, \xi_{j}\right), i \neq j$, $i, j=1,2, \ldots, 8$ are circles, ellipses, segments and points, so it is a plausible counterpart of the periodic evolution of the qubit.

\subsection{The case with $a * a=-|a| a$}

It can be easily checked that the solution to (3.1) in the case of $\boldsymbol{a} * \boldsymbol{a}=-|\boldsymbol{a}| \boldsymbol{a}$ can be obtained from the solution (3.19) to (3.1) with $\boldsymbol{a} * \boldsymbol{a}=|\boldsymbol{a}| \boldsymbol{a}$ by the formal replacement $\boldsymbol{a} \rightarrow-\boldsymbol{a}$ and $t \rightarrow-t$. Hence, we get for $\boldsymbol{a} * \boldsymbol{a}=-|\boldsymbol{a}| \boldsymbol{a}$

$$
\begin{aligned}
\boldsymbol{\xi}(t)= & \left(\frac{1}{3}+\frac{2}{3} \cos (t \sqrt{3}|\boldsymbol{a}|)\right) \boldsymbol{\xi}_{0}+\frac{2}{3|\boldsymbol{a}|} \sin (t \sqrt{3}|\boldsymbol{a}|) \boldsymbol{a} \wedge \boldsymbol{\xi}_{0} \\
& +(1-\cos (t \sqrt{3}|\boldsymbol{a}|))\left(\frac{2}{3|\boldsymbol{a}|} \boldsymbol{a} * \boldsymbol{\xi}_{0}+\frac{4}{3 \boldsymbol{a}^{2}}\left(\boldsymbol{a} \cdot \boldsymbol{\xi}_{0}\right) \boldsymbol{a}\right) .
\end{aligned}
$$


Analogously, applying the transformation $\boldsymbol{a} \rightarrow-\boldsymbol{a}$ to (3.20), (3.21) and (3.22) we arrive at the stationary solutions of the form:

$$
\begin{aligned}
\bar{\xi} & =-\frac{\boldsymbol{a}}{|\boldsymbol{a}|} \quad \text { (pure state) } \\
\overline{\boldsymbol{\xi}}^{\prime} & =\frac{\boldsymbol{a}}{2|\boldsymbol{a}|} \quad \text { (mixed state on the boundary of the state space), } \\
\bar{\xi}^{\prime \prime} & =\frac{\boldsymbol{a}}{\sigma|\boldsymbol{a}|}, \quad \sigma>2 \quad \text { (mixed state in the interior of the space of states). }
\end{aligned}
$$

It is clear that qualitative behavior of solutions to (3.1) given by (3.23) is the same as in the case with $\boldsymbol{a} * \boldsymbol{a}=|\boldsymbol{a}| \boldsymbol{a}$.

\subsection{The condition $a \cdot(a * a)=0$}

Using the system of recurrence equations (3.3) for $\boldsymbol{a} \cdot(\boldsymbol{a} * \boldsymbol{a})=0$, we easily find

$$
\mathrm{e}^{\tau \boldsymbol{a} \cdot \boldsymbol{\lambda}}=\frac{1}{3}+\frac{2}{3} \cosh (\tau|\boldsymbol{a}|)+\left[\frac{\sinh (\tau|\boldsymbol{a}|)}{|\boldsymbol{a}|} \boldsymbol{a}+\frac{1}{\sqrt{3} \boldsymbol{a}^{2}}(\cosh (\tau|\boldsymbol{a}|)-1) \boldsymbol{a} * \boldsymbol{a}\right] \cdot \boldsymbol{\lambda} .
$$

On setting $\tau=-i t$ and using (1.1), (2.7) and (2.8), we obtain after some calculation the solution to (3.1) such that

$$
\begin{aligned}
\boldsymbol{\xi}(t)= & \frac{1}{3}\left(2 \cos ^{2}(t|\boldsymbol{a}|)+2 \cos (t|\boldsymbol{a}|)-1\right) \boldsymbol{\xi}_{0}+\frac{2}{3 \sqrt{3}|\boldsymbol{a}|}(2 \cos (t|\boldsymbol{a}|)+1) \sin (t|\boldsymbol{a}|) \boldsymbol{a} \wedge \boldsymbol{\xi}_{0} \\
& +\frac{2}{3 \boldsymbol{a}^{2}}(2 \cos (t|\boldsymbol{a}|)+1)(\cos (t|\boldsymbol{a}|)-1)(\boldsymbol{a} * \boldsymbol{a}) * \boldsymbol{\xi}_{0}+2 \frac{\sin ^{2}(t|\boldsymbol{a}|)}{\boldsymbol{a}^{2}}\left(\boldsymbol{a} \cdot \boldsymbol{\xi}_{0}\right) \boldsymbol{a} \\
& +\frac{2}{3|\boldsymbol{a}|^{4}}(\cos (t|\boldsymbol{a}|)-1)^{2}\left[(\boldsymbol{a} * \boldsymbol{a}) \cdot \boldsymbol{\xi}_{0}\right] \boldsymbol{a} * \boldsymbol{a}-\frac{2}{3 \sqrt{3}|\boldsymbol{a}|^{3}}(\cos (t|\boldsymbol{a}|)-1) \sin (t|\boldsymbol{a}|) \\
& \times\left\{\boldsymbol{a} *\left[(\boldsymbol{a} * \boldsymbol{a}) \wedge \boldsymbol{\xi}_{0}\right]-(\boldsymbol{a} * \boldsymbol{a}) *\left(\boldsymbol{a} \wedge \boldsymbol{\xi}_{0}\right)\right\} .
\end{aligned}
$$

Now, (3.15) implies the following form of the stationary solutions to (3.1) referring to the pure states:

$$
\overline{\boldsymbol{\xi}}_{1}=-\frac{\boldsymbol{a} * \boldsymbol{a}}{\boldsymbol{a}^{2}}, \quad \overline{\boldsymbol{\xi}}_{2,3}= \pm \frac{\sqrt{3}}{2|\boldsymbol{a}|} \boldsymbol{a}+\frac{1}{2 \boldsymbol{a}^{2}} \boldsymbol{a} * \boldsymbol{a} .
$$

Further, setting $v=0$ and $\boldsymbol{a}^{2} \mu^{2}+|\boldsymbol{a}|^{4} v^{2}=\frac{1}{3}$ in (3.16) with $\boldsymbol{a} \cdot(\boldsymbol{a} * \boldsymbol{a})=0$, we obtain the following stationary solutions corresponding to the mixed states on the boundary of the space of states: 


$$
\bar{\xi}= \pm \frac{a}{\sqrt{3}|\boldsymbol{a}|}, \quad(v=0)
$$

and

$$
\overline{\boldsymbol{\xi}}_{1,2}=\frac{\boldsymbol{a}}{2 \sqrt{3}|\boldsymbol{a}|} \pm \frac{\boldsymbol{a} * \boldsymbol{a}}{2 \boldsymbol{a}^{2}}, \quad \overline{\boldsymbol{\xi}}_{3,4}=-\frac{\boldsymbol{a}}{2 \sqrt{3}|\boldsymbol{a}|} \pm \frac{\boldsymbol{a} * \boldsymbol{a}}{2 \boldsymbol{a}^{2}} \quad\left(\boldsymbol{a}^{2} \mu^{2}+|\boldsymbol{a}|^{4} v^{2}=\frac{1}{3}\right)
$$

Finally, assuming that $\boldsymbol{a}^{2} \mu^{2}+|\boldsymbol{a}|^{4} v^{2}=\frac{1}{3}$ we find the stationary solutions (3.13) referring to mixed states in the interior of the space of states specified by

$$
\mu= \pm \sqrt{\frac{1}{3}-|\boldsymbol{a}|^{4} v^{2}}, \quad 0<v<\frac{1}{2 \boldsymbol{a}^{2}}
$$

\subsection{Diagonal generator of evolution $a \cdot \lambda$}

We now discuss the case of the diagonal matrix $\boldsymbol{a} \cdot \boldsymbol{\lambda}$. Since the only diagonal GellMann matrices are $\lambda_{3}$ and $\lambda_{8}$, we then have $\boldsymbol{a}=\left(0,0, a_{3}, 0,0,0,0, a_{8}\right)$. We point out that since $\boldsymbol{a} * \boldsymbol{a}=\left(0,0,2 a_{3} a_{8}, 0,0,0,0, a_{3}^{2}-a_{8}^{2}\right)$, the vector $\boldsymbol{a}$ satisfies the condition $\boldsymbol{a} * \boldsymbol{a}=|\boldsymbol{a}| \boldsymbol{a}$ for $a_{3}=0$ and $a_{8} \leqslant 0$, and for $a_{3}= \pm \sqrt{3} a_{8}$ and $a_{8}>0$, so we then have the case discussed in Sect. 3.1. Analogously, for $a_{3}=0$ and $a_{8} \geqslant 0$ as well as for $a_{3}= \pm \sqrt{3} a_{8}$ and $a_{8}<0$, we get the condition $\boldsymbol{a} * \boldsymbol{a}=-|\boldsymbol{a}| \boldsymbol{a}$ and we then deal wit the case investigated in Sect. 3.2. Furthermore, for $a_{8}=0$ and the condition $a_{8} \neq 0$ and $a_{3}= \pm \frac{1}{\sqrt{3}} a_{8}$, we have $\boldsymbol{a} \cdot(\boldsymbol{a} * \boldsymbol{a})=0$, so we then have the case analyzed in Sect. 3.3.

Now, it can be easily checked that

$$
\mathrm{e}^{\tau\left(a_{3} \lambda_{3}+a_{8} \lambda_{8}\right)}=f(\tau)+\gamma(\tau) \lambda_{3}+\delta(\tau) \lambda_{8},
$$

where

$$
\begin{aligned}
& f(\tau)=\frac{1}{3}\left[\mathrm{e}^{\left.\tau\left(a_{3}+\frac{1}{\sqrt{3}}\right) a_{8}\right)}+\mathrm{e}^{\left.\tau\left(-a_{3}+\frac{1}{\sqrt{3}}\right) a_{8}\right)}+\mathrm{e}^{-2 \tau \frac{a_{8}}{\sqrt{3}}}\right], \\
& \gamma(\tau)=\frac{1}{2}\left[\mathrm{e}^{\left.\tau\left(a_{3}+\frac{1}{\sqrt{3}}\right) a_{8}\right)}-\mathrm{e}^{\left.\tau\left(-a_{3}+\frac{1}{\sqrt{3}}\right) a_{8}\right)}\right], \\
& \delta(\tau)=\frac{\sqrt{3}}{6}\left[\mathrm{e}^{\left.\tau\left(a_{3}+\frac{1}{\sqrt{3}}\right) a_{8}\right)}+\mathrm{e}^{\left.\tau\left(-a_{3}+\frac{1}{\sqrt{3}}\right) a_{8}\right)}-2 \mathrm{e}^{-2 \tau \frac{a_{8}}{\sqrt{3}}}\right] .
\end{aligned}
$$

On setting $\tau=-i t$ and making use of (1.1), (2.7) and (2.8), we arrive after some calculation at the following solution of (3.1):

$$
\begin{aligned}
& \xi_{1}(t)=\cos \left(2 t a_{3}\right) \xi_{01}-\sin \left(2 t a_{3}\right) \xi_{02} \\
& \xi_{2}(t)=\sin \left(2 t a_{3}\right) \xi_{01}+\cos \left(2 t a_{3}\right) \xi_{02}, \\
& \xi_{3}(t)=\xi_{03}
\end{aligned}
$$



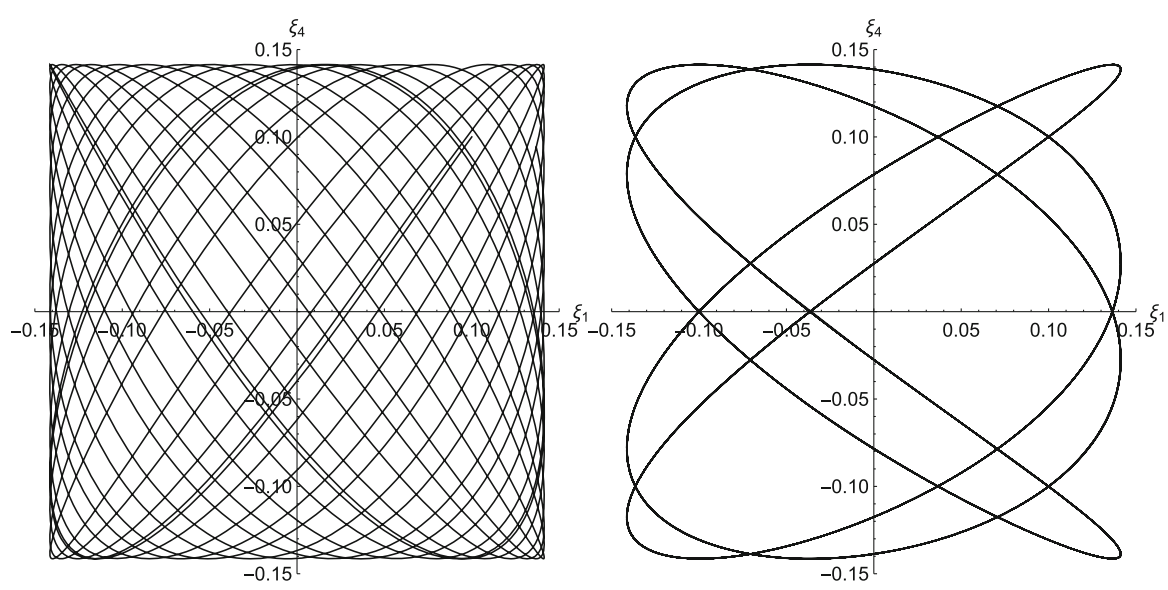

Fig. 3 Trajectories of the system (3.1) given by (3.37) corresponding to the diagonal operator of evolution. Left: the quasiperiodic trajectory specified by $\boldsymbol{a}=\left(0,0, \frac{1}{2}, 0,0,0,0, \frac{1}{2}\right)$ and $\xi_{0}=(0.1,0.1,0.1,0.1,0.1,0.1,0.1,0.1)$ for $t=100$. The projection of the trajectory onto the $\left(\xi_{1}, \xi_{4}\right)$ plane. Right: the periodic trajectory referring to $\boldsymbol{a}=\left(0,0, \sqrt{3}, 0,0,0,0, \frac{1}{2}\right)$ and $\xi_{0}=$ $(0.1,0.1,0.1,0.1,0.1,0.1,0.1,0.1)$

$$
\begin{aligned}
& \xi_{4}(t)=\cos \left[t\left(a_{3}+\sqrt{3} a_{8}\right)\right] \xi_{04}-\sin \left[t\left(a_{3}+\sqrt{3} a_{8}\right)\right] \xi_{05}, \\
& \xi_{5}(t)=\sin \left[t\left(a_{3}+\sqrt{3} a_{8}\right)\right] \xi_{04}+\cos \left[t\left(a_{3}+\sqrt{3} a_{8}\right)\right] \xi_{05}, \\
& \xi_{6}(t)=\cos \left[t\left(a_{3}-\sqrt{3} a_{8}\right)\right] \xi_{06}+\sin \left[t\left(a_{3}-\sqrt{3} a_{8}\right)\right] \xi_{07}, \\
& \xi_{7}(t)=-\sin \left[t\left(a_{3}-\sqrt{3} a_{8}\right)\right] \xi_{06}+\cos \left[t\left(a_{3}-\sqrt{3} a_{8}\right)\right] \xi_{07}, \\
& \xi_{8}(t)=\xi_{08} .
\end{aligned}
$$

The solution (3.37) is in general three-frequency quasiperiodic solution. This observation is consistent with the general formula (3.12) for the exponential of $\boldsymbol{a} \cdot \boldsymbol{\lambda}$. Examples of the trajectories given by (3.37) that are the Lissajous curves are illustrated in Fig. 3.

We now discuss the stationary solutions. From (3.13) and (A.10), it follows that the stationary solutions in the discussed case are of the form $\bar{\xi}=\left(0,0, \bar{\xi}_{3}, 0,0,0,0, \bar{\xi}_{8}\right)$. Hence, we find that the stationary solutions referring to the pure states are $\bar{\xi}_{1}=(0,0,0,0,0,0,0,-1)$ and $\bar{\xi}_{2,3}=\left(0,0, \pm \frac{\sqrt{3}}{2}, 0,0,0,0, \frac{1}{2}\right)$. The stationary solutions corresponding to the mixed states on the boundary of the state space can be easily obtained from (2.5). Namely, we have

$$
\begin{aligned}
\bar{\xi}_{8}= & \frac{1}{2} \sqrt[3]{\frac{1}{2}(1-3 \kappa)+\sqrt{-\kappa^{3}+\frac{1}{4}(1-3 \kappa)^{2}}} \\
& +\frac{1}{2} \sqrt[3]{\frac{1}{2}(1-3 \kappa)-\sqrt{-\kappa^{3}+\frac{1}{4}(1-3 \kappa)^{2}}} \\
\bar{\xi}_{3 ; 1,2}= & \pm \sqrt{\kappa-\bar{\xi}_{8}^{2}}, \quad \kappa \in\left(0, \frac{1}{4}\right],
\end{aligned}
$$




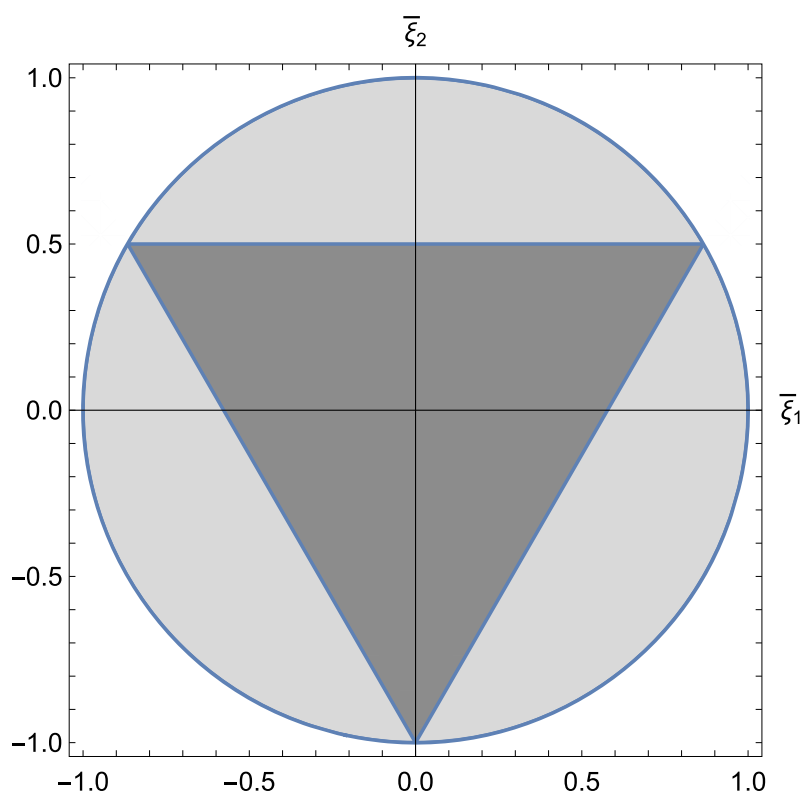

Fig. 4 The region in the plane (darker triangle) containing stationary solutions of (3.1) corresponding to mixed states in the interior of the state space, given by (3.40) (the case of the diagonal generator of evolution). The vertices of the triangle $\left(-\frac{\sqrt{3}}{2}, \frac{1}{2}\right),(0,-1)$, and $\left(\frac{\sqrt{3}}{2}, \frac{1}{2}\right)$ refer to pure states and its edges to mixed states on the boundary of the space of states

where $\kappa$ is the parameter defined by $\bar{\xi}_{3}^{2}+\bar{\xi}_{8}^{2}=\kappa$, so in the general case of the mixed states on the boundary it satisfies $\kappa \in(0,1)$. In the particular case of $\kappa=\frac{1}{4}$, the relations (3.38) reduce to $\bar{\xi}_{8}=\frac{1}{2}$ and $\bar{\xi}_{3}=0$. Further, the trigonometric solution to the cubic equation satisfied by $\bar{\xi}_{8}$ results in

$$
\begin{aligned}
\bar{\xi}_{8,1}=\sqrt{\kappa} \cos \frac{\alpha}{3}, & \bar{\xi}_{8 ; 2,3}=-\sqrt{\kappa} \cos \left(\frac{\alpha}{3} \pm \frac{\pi}{3}\right), \\
\bar{\xi}_{3 ; 1,2}= \pm \sqrt{\kappa-\bar{\xi}_{8}^{2}}, & \kappa \in\left(\frac{1}{4}, 1\right),
\end{aligned}
$$

where $\cos \alpha=-4 \frac{3 \kappa-1}{\sqrt{\kappa^{3}}}$. For $\kappa=\frac{1}{3}$, formula (3.39) simplifies to $\bar{\xi}_{8,1}=\frac{1}{2}, \bar{\xi}_{8,2}=0$, $\bar{\xi}_{8,3}=-\frac{1}{2}$, corresponding to $\bar{\xi}_{3 ; 1,3}= \pm \frac{1}{2 \sqrt{3}}, \bar{\xi}_{3,2}= \pm \frac{1}{\sqrt{3}}$, respectively. The stationary solutions referring to the mixed states in the interior of the state space are specified by the system of the inequalities such that (see (2.2))

$$
\left\{\begin{array}{l}
\bar{\xi}_{3}^{2}+\bar{\xi}_{8}^{2}<1, \\
2 \bar{\xi}_{8}^{3}-6 \bar{\xi}_{3}^{2} \bar{\xi}_{8}+3\left(\bar{\xi}_{3}^{2}+\bar{\xi}_{8}^{2}\right)<1 .
\end{array}\right.
$$

The region in the plane defined by (3.40) is depicted in Fig. 4. The vertices of the triangle correspond to the pure states, and its edges refer to the mixed states on the 
boundary of the space of states. The parametrization of edges is given by (3.38) and (3.39). We finally point out that the case of the diagonal Gell-Mann matrices was analyzed in the context of the properties of the states of qutrits in $[16,17]$.

\section{Nonlinear evolution of a qutrit}

We first confine ourselves to the case of $\boldsymbol{a}=0$, i.e., $H=0$. The system (2.9) takes then the form

$$
\dot{\xi}=\frac{2}{\sqrt{3}} \boldsymbol{b}+\frac{2}{\sqrt{3}} \boldsymbol{b} * \boldsymbol{\xi}-\frac{4}{\sqrt{3}}(\boldsymbol{b} \cdot \boldsymbol{\xi}) \boldsymbol{\xi}, \quad \boldsymbol{\xi}(0)=\boldsymbol{\xi}_{0} .
$$

\subsection{The condition $b * b=|b| b$}

Proceeding analogously as with (3.1), we first consider the case with $\boldsymbol{b} * \boldsymbol{b}=|\boldsymbol{b}| \boldsymbol{b}$. Taking into account (3.18) with $\tau=t,(1.1),(2.7)$ and (2.8), we get the solution to (4.1) of the form

$$
\xi(t)=\frac{\eta(t)}{\varphi(t)},
$$

where

$$
\begin{aligned}
\eta(t)= & \left(-\frac{1}{9} \mathrm{e}^{4 t \frac{\sqrt{3}}{3}|\boldsymbol{b}|}+\frac{8}{9} \mathrm{e}^{t \frac{\sqrt{3}}{3}|\boldsymbol{b}|}+\frac{2}{9} \mathrm{e}^{-2 t \frac{\sqrt{3}}{3}|\boldsymbol{b}|}\right) \boldsymbol{\xi}_{0} \\
& +\frac{1}{9|\boldsymbol{b}|}\left(\mathrm{e}^{4 t \frac{\sqrt{3}}{3}|\boldsymbol{b}|}+4 \mathrm{e}^{t \frac{\sqrt{3}}{3}|\boldsymbol{b}|}-5 \mathrm{e}^{-2 t \frac{\sqrt{3}}{3}|\boldsymbol{b}|}\right) \boldsymbol{b} * \boldsymbol{\xi}_{0} \\
& +\frac{4}{9 \boldsymbol{b}^{2}}\left(\mathrm{e}^{4 t \frac{\sqrt{3}}{3}|\boldsymbol{b}|}-2 \mathrm{e}^{t \frac{\sqrt{3}}{3}|\boldsymbol{b}|}+\mathrm{e}^{-2 t \frac{\sqrt{3}}{3}|\boldsymbol{b}|}\right)\left(\boldsymbol{b} \cdot \boldsymbol{\xi}_{0}\right) \boldsymbol{b} \\
& +\frac{2}{9 \boldsymbol{b}^{2}}\left(\mathrm{e}^{4 t \frac{\sqrt{3}}{3}|\boldsymbol{b}|}-2 \mathrm{e}^{t \frac{\sqrt{3}}{3}|\boldsymbol{b}|}+\mathrm{e}^{-2 t \frac{\sqrt{3}}{3}|\boldsymbol{b}|}\right)\left(\boldsymbol{b} * \boldsymbol{\xi}_{0}\right) * \boldsymbol{b} \\
& +\frac{1}{3|\boldsymbol{b}|}\left(\mathrm{e}^{4 t \frac{\sqrt{3}}{3}|\boldsymbol{b}|}-\mathrm{e}^{-2 t \frac{\sqrt{3}}{3}|\boldsymbol{b}|}\right) \boldsymbol{b}, \\
\varphi(t)= & \frac{1}{3} \mathrm{e}^{4 t \frac{\sqrt{3}}{3}|\boldsymbol{b}|}+\frac{2}{3} \mathrm{e}^{-2 t \frac{\sqrt{3}}{3}|\boldsymbol{b}|}+\frac{2}{3|\boldsymbol{b}|}\left(\mathrm{e}^{4 t \frac{\sqrt{3}}{3}|\boldsymbol{b}|}-\mathrm{e}^{-2 t \frac{\sqrt{3}}{3}|\boldsymbol{b}|}\right) \boldsymbol{b} \cdot \boldsymbol{\xi}_{0} .
\end{aligned}
$$

Taking into account (4.1), one can easily check that we have in the discussed case the following stationary solution:

$$
\bar{\xi}=\frac{b}{|b|} .
$$

It follows from numerical calculations that the stationary solution (4.5) is asymptotically stable. An easy example of solution to (4.1) going to $\bar{\xi}$ as $t \rightarrow \infty$ can be 
obtained from (4.4) by setting $\xi_{0}=0$. The problem of finding the stationary solutions to (4.1) that are not asymptotically stable seems to be a difficult task. Such solution proportional to $\boldsymbol{b}$ is of the form

$$
\bar{\xi}^{\prime}=-\frac{b}{2|\boldsymbol{b}|}
$$

It can be easily verified that (4.5) and (4.6) represent the pure state and the mixed state on the boundary of the state space, respectively.

\subsection{The case of $b * b=-|b| b$}

Proceeding analogously as in Sect. 3.2 and replacing in (4.3) $\boldsymbol{b}$ with $-\boldsymbol{b}$ and $t$ with $-t$, we find that the solution to (4.1) corresponding to $\boldsymbol{b} * \boldsymbol{b}=-|\boldsymbol{b}| \boldsymbol{b}$ is given by (4.2), where

$$
\begin{aligned}
\boldsymbol{\eta}(t)= & \left(\frac{2}{9} \mathrm{e}^{2 t \frac{\sqrt{3}}{3}|\boldsymbol{b}|}+\frac{8}{9} \mathrm{e}^{-t \frac{\sqrt{3}}{3}|\boldsymbol{b}|}-\frac{1}{9} \mathrm{e}^{-4 t \frac{\sqrt{3}}{3}|\boldsymbol{b}|}\right) \boldsymbol{\xi}_{0} \\
& +\frac{1}{9|\boldsymbol{b}|}\left(5 \mathrm{e}^{2 t \frac{\sqrt{3}}{3}|\boldsymbol{b}|}-4 \mathrm{e}^{-t \frac{\sqrt{3}}{3}|\boldsymbol{b}|}-\mathrm{e}^{-4 t \frac{\sqrt{3}}{3}|\boldsymbol{b}|}\right) \boldsymbol{b} * \boldsymbol{\xi}_{0} \\
& +\frac{4}{9 \boldsymbol{b}^{2}}\left(\mathrm{e}^{2 t \frac{\sqrt{3}}{3}|\boldsymbol{b}|}-2 \mathrm{e}^{-t \frac{\sqrt{3}}{3}|\boldsymbol{b}|}+\mathrm{e}^{-4 t \frac{\sqrt{3}}{3}|\boldsymbol{b}|}\right)\left(\boldsymbol{b} \cdot \boldsymbol{\xi}_{0}\right) \boldsymbol{b} \\
& +\frac{2}{9 \boldsymbol{b}^{2}}\left(\mathrm{e}^{2 t \frac{\sqrt{3}}{3}|\boldsymbol{b}|}-2 \mathrm{e}^{-t \frac{\sqrt{3}}{3}|\boldsymbol{b}|}+\mathrm{e}^{-4 t \frac{\sqrt{3}}{3}|\boldsymbol{b}|}\right)\left(\boldsymbol{b} * \boldsymbol{\xi}_{0}\right) * \boldsymbol{b} \\
& +\frac{1}{3|\boldsymbol{b}|}\left(\mathrm{e}^{2 t \frac{\sqrt{3}}{3}|\boldsymbol{b}|}-\mathrm{e}^{-4 t \frac{\sqrt{3}}{3}|\boldsymbol{b}|}\right) \boldsymbol{b}, \\
\varphi(t)= & \frac{2}{3} \mathrm{e}^{2 t \frac{\sqrt{3}}{3}|\boldsymbol{b}|}+\frac{1}{3} \mathrm{e}^{-4 t \frac{\sqrt{3}}{3}|\boldsymbol{b}|}+\frac{2}{3|\boldsymbol{b}|}\left(\mathrm{e}^{2 t \frac{\sqrt{3}}{3}|\boldsymbol{b}|}-\mathrm{e}^{-4 t \frac{\sqrt{3}}{3}|\boldsymbol{b}|}\right) \boldsymbol{b} \cdot \boldsymbol{\xi}_{0} .
\end{aligned}
$$

The system (4.1) has in the discussed case the stationary solution such that

$$
\bar{\xi}=\frac{b}{2|\boldsymbol{b}|} .
$$

The numerical simulations indicate that the stationary solution (4.9) is asymptotically stable. The solution (4.9) represents the mixed state on the boundary of the space of states. We point out that there are no asymptotically stable stationary solutions representing mixed states in the case of the qubit [1]. The asymptotically stable stationary solution (4.9) is not globally stable. Consider for example the case of $\boldsymbol{b}=\left(0,0, \frac{\sqrt{3}}{2}, 0,0,0,0,-\frac{1}{2}\right)$. It appears that the mixed states on the boundary of the state space $\xi_{0}=\left(0,0, \frac{1}{2}, 0,0,0,0, \frac{1}{2}\right)$ and $\xi_{0}=\left(0,0, \frac{1}{2}(1-\right.$ $\sqrt{3}), 0,0,0,0, \frac{1}{2}(1-\sqrt{3})$ ) approach the stationary solutions representing the pure states $\bar{\xi}=\left(0,0, \frac{\sqrt{3}}{2}, 0,0,0,0,-\frac{1}{2}\right)$ and $\bar{\xi}=(0,0,0,0,0,0,0,-1)$, respectively. Furthermore, we have the stationary solution to (4.1) that is not asymptotically stable of the form 


$$
\bar{\xi}^{\prime}=-\frac{b}{|\boldsymbol{b}|} .
$$

The solution (4.10) represents the pure state. It is clear that it is not the unique one.

\subsection{The condition $b \cdot(b * b)=0$}

We now discuss the case that is the nonlinear counterpart of the condition investigated in Sect. 3.3. Applying the algorithm used for calculation of (3.28), we arrive at the following solution to (4.1) given by (4.2) and the relations

$$
\begin{aligned}
\boldsymbol{\eta}(t)= & \frac{1}{3}(2 \cosh (t|\boldsymbol{b}|)+1) \boldsymbol{\xi}_{0}+\frac{2}{\sqrt{3}} \frac{\sinh (t|\boldsymbol{b}|)}{|\boldsymbol{b}|} \boldsymbol{b} * \boldsymbol{\xi}_{0}+\frac{2}{3 \boldsymbol{b}^{2}}\left(1-\cosh (t|\boldsymbol{b}|)(\boldsymbol{b} * \boldsymbol{b}) * \boldsymbol{\xi}_{0}\right. \\
& +2 \frac{\sinh (t|\boldsymbol{b}|)}{|\boldsymbol{b}|}\left[\frac{\sinh (t|\boldsymbol{b}|)}{|\boldsymbol{b}|} \boldsymbol{b} \cdot \boldsymbol{\xi}_{0}+\frac{1}{\sqrt{3} \boldsymbol{b}^{2}}(\cosh (t|\boldsymbol{b}|)-1)(\boldsymbol{b} * \boldsymbol{b}) \cdot \boldsymbol{\xi}_{0}\right] \boldsymbol{b} \\
& +\frac{2}{\sqrt{3} \boldsymbol{b}^{2}}(\cosh (t|\boldsymbol{b}|)-1)\left[\frac{\sinh (t|\boldsymbol{b}|)}{|\boldsymbol{b}|} \boldsymbol{b} \cdot \boldsymbol{\xi}_{0}+\frac{1}{\sqrt{3} \boldsymbol{b}^{2}}(\cosh (t|\boldsymbol{b}|)-1)(\boldsymbol{b} * \boldsymbol{b}) \cdot \boldsymbol{\xi}_{0}\right] \\
& \times \boldsymbol{b} * \boldsymbol{b}+\frac{1}{\sqrt{3}} \frac{\sinh (2 t|\boldsymbol{b}|)}{|\boldsymbol{b}|} \boldsymbol{b}+\frac{1}{3 \boldsymbol{b}^{2}}(\cosh (2 t|\boldsymbol{b}|)-1) \boldsymbol{b} * \boldsymbol{b}, \\
\varphi(t)= & \frac{2}{3} \cosh (2 t|\boldsymbol{b}|)+\frac{1}{3}+\frac{2 \sqrt{3}}{3} \frac{\sinh (2 t|\boldsymbol{b}|)}{|\boldsymbol{b}|} \boldsymbol{b} \cdot \boldsymbol{\xi}_{0}+\frac{2}{3 \boldsymbol{b}^{2}}(\cosh (2 t|\boldsymbol{b}|)-1)(\boldsymbol{b} * \boldsymbol{b}) \cdot \boldsymbol{\xi}_{0} .
\end{aligned}
$$

On making the ansatz of the form (3.13) with $\boldsymbol{a}$ replaced by $\boldsymbol{b}$, one can easily obtain the following stationary solutions to (4.1):

$$
\begin{aligned}
\bar{\xi} & =\frac{\sqrt{3}}{2|\boldsymbol{b}|} \boldsymbol{b}+\frac{\boldsymbol{b} * \boldsymbol{b}}{2 \boldsymbol{b}^{2}}, \\
\bar{\xi}^{\prime} & =-\frac{\sqrt{3}}{2|\boldsymbol{b}|} \boldsymbol{b}+\frac{\boldsymbol{b} * \boldsymbol{b}}{2 \boldsymbol{b}^{2}}, \\
\bar{\xi}^{\prime \prime} & =-\frac{\boldsymbol{b} * \boldsymbol{b}}{\boldsymbol{b}^{2}} .
\end{aligned}
$$

All the stationary solutions represent the pure states. The numerical calculations show that the solution $\bar{\xi}$ is asymptotically stable and the solutions $\bar{\xi}^{\prime}$ and $\bar{\xi}^{\prime \prime}$ are unstable.

\subsection{Diagonal matrix $b \cdot \lambda$}

We now study the case of $\boldsymbol{b} \cdot \lambda=b_{3} \lambda_{3}+b_{8} \lambda_{8}$, that is, the counterpart of the diagonal generator of the unitary evolution analyzed in Sect. 3.4. Using (3.33) with $\tau$ replaced by $t$, we find after some algebra the following solution to (4.1): 


$$
\begin{aligned}
\eta_{1}(t)= & \mathrm{e}^{2 t \frac{b_{8}}{\sqrt{3}}} \xi_{01}, \\
\eta_{2}(t)= & \mathrm{e}^{2 t \frac{b_{8}}{\sqrt{3}} \xi_{02},} \\
\eta_{3}(t)= & \frac{1}{2}\left[\mathrm{e}^{2 t\left(b_{3}+\frac{1}{\sqrt{3}} b_{8}\right)}+\mathrm{e}^{\left.2 t\left(-b_{3}+\frac{1}{\sqrt{3}} b_{8}\right)\right] \xi_{03}+f_{38}(t) \xi_{08}}\right. \\
& +\frac{1}{2 \sqrt{3}}\left[\mathrm{e}^{2 t\left(b_{3}+\frac{1}{\sqrt{3}} b_{8}\right)}-\mathrm{e}^{2 t\left(-b_{3}+\frac{1}{\sqrt{3}} b_{8}\right)}\right], \\
\eta_{4}(t)= & f_{45}(t) \xi_{04}, \\
\eta_{5}(t)= & f_{45}(t) \xi_{05}, \\
\eta_{6}(t)= & f_{67}(t) \xi_{06}, \\
\eta_{7}(t)= & f_{67}(t) \xi_{07}, \\
\eta_{8}(t)= & f_{38}(t) \xi_{03}+\left\{\frac{1}{6}\left[\mathrm{e}^{2 t\left(b_{3}+\frac{1}{\sqrt{3}} b_{8}\right)}+\mathrm{e}^{2 t\left(-b_{3}+\frac{1}{\sqrt{3}} b_{8}\right)}\right]+\frac{2}{3} \mathrm{e}^{-4 t \frac{b_{8}}{\sqrt{3}}}\right\} \xi_{08} \\
& +\frac{1}{6}\left[\mathrm{e}^{2 t\left(b_{3}+\frac{1}{\sqrt{3}} b_{8}\right)}+\mathrm{e}^{2 t\left(-b_{3}+\frac{1}{\sqrt{3}} b_{8}\right)}-2 \mathrm{e}^{-4 t \frac{b_{8}}{\sqrt{3}}}\right],
\end{aligned}
$$

where

$$
\begin{aligned}
f_{38}(t)= & \frac{1}{9}\left[(2 \sqrt{3}-1) \mathrm{e}^{2 t\left(b_{3}+\frac{1}{\sqrt{3}} b_{8}\right)}-(2 \sqrt{3}+1) \mathrm{e}^{2 t\left(-b_{3}+\frac{1}{\sqrt{3}} b_{8}\right)}\right. \\
& +(-\sqrt{3}+1) \mathrm{e}^{t\left(b_{3}-\frac{1}{\sqrt{3}} b_{8}\right)}+(\sqrt{3}+1) \mathrm{e}^{t\left(-b_{3}-\frac{1}{\sqrt{3}} b_{8}\right)}+\mathrm{e}^{2 t \frac{1}{\sqrt{3}} b_{8}}-\mathrm{e}^{\left.-4 t \frac{1}{\sqrt{3}} b_{8}\right]}, \\
f_{45}(t)= & \left(\frac{1}{12}-\frac{\sqrt{3}}{18}\right) \mathrm{e}^{2 t\left(b_{3}+\frac{1}{\sqrt{3}} b_{8}\right)}-\left(\frac{1}{12}+\frac{\sqrt{3}}{18}\right) \mathrm{e}^{2 t\left(-b_{3}+\frac{1}{\sqrt{3}} b_{8}\right)} \\
& +\left(\frac{5}{6}+\frac{\sqrt{3}}{18}\right) \mathrm{e}^{t\left(b_{3}-\frac{1}{\sqrt{3}} b_{8}\right)}+\left(\frac{1}{6}+\frac{\sqrt{3}}{18}\right) \mathrm{e}^{t\left(-b_{3}-\frac{1}{\sqrt{3}} b_{8}\right)} \\
& +\frac{\sqrt{3}}{18}\left(\mathrm{e}^{2 t \frac{1}{\sqrt{3}} b_{8}}-\mathrm{e}^{-4 t \frac{1}{\sqrt{3}} b_{8}}\right), \\
& \left(-\frac{1}{12}+\frac{\sqrt{3}}{18}\right) \mathrm{e}^{2 t\left(b_{3}+\frac{1}{\sqrt{3}} b_{8}\right)}+\left(\frac{1}{12}+\frac{\sqrt{3}}{18}\right) \mathrm{e}^{2 t\left(-b_{3}+\frac{1}{\sqrt{3}} b_{8}\right)} \\
f_{67}(t)= & \left(\frac{1}{6}-\frac{\sqrt{3}}{18}\right) \mathrm{e}^{t\left(b_{3}-\frac{1}{\sqrt{3}} b_{8}\right)}+\left(\frac{5}{6}-\frac{\sqrt{3}}{18}\right) \mathrm{e}^{t\left(-b_{3}-\frac{1}{\sqrt{3}} b_{8}\right)} \\
& -\frac{\sqrt{3}}{18}\left(\mathrm{e}^{2 t \frac{1}{\sqrt{3}} b_{8}}-\mathrm{e}^{-4 t \frac{1}{\sqrt{3}} b_{8}}\right) \cdot
\end{aligned}
$$




$$
\begin{aligned}
\varphi(t)= & \frac{1}{3}\left(\mathrm{e}^{2 t\left(b_{3}+\frac{1}{\sqrt{3}} b_{8}\right)}+\mathrm{e}^{2 t\left(-b_{3}+\frac{1}{\sqrt{3}} b_{8}\right)}+\mathrm{e}^{-4 t \frac{1}{\sqrt{3}} b_{8}}\right) \\
& +\frac{\sqrt{3}}{3}\left(\mathrm{e}^{2 t\left(b_{3}+\frac{1}{\sqrt{3}} b_{8}\right)}-\mathrm{e}^{2 t\left(-b_{3}+\frac{1}{\sqrt{3}} b_{8}\right)}\right) \xi_{03} \\
& +\frac{1}{3}\left(\mathrm{e}^{2 t\left(b_{3}+\frac{1}{\sqrt{3}} b_{8}\right)}+\mathrm{e}^{2 t\left(-b_{3}+\frac{1}{\sqrt{3}} b_{8}\right)}-2 \mathrm{e}^{-4 t \frac{1}{\sqrt{3}} b_{8}}\right) \xi_{08} .
\end{aligned}
$$

As mentioned earlier in Sect. 3.4, $b_{3}= \pm \sqrt{3} b_{8}$ and $b_{8}>0$ correspond to $\boldsymbol{b} * \boldsymbol{b}=|\boldsymbol{b}| \boldsymbol{b}$; $b_{3}=0$ and $b_{8} \geqslant 0$ as well as $b_{3}= \pm \sqrt{3} b_{8}$ and $b_{8}<0$ refer to $\boldsymbol{b} * \boldsymbol{b}=-|\boldsymbol{b}| \boldsymbol{b}$. Furthermore, $b_{8}=0$ and the conditions $b_{3}= \pm \frac{1}{\sqrt{3}} a_{8}$ and $b_{8} \neq 0$ lead to $\boldsymbol{b} \cdot(\boldsymbol{b} * \boldsymbol{b})=0$. Of course, in such cases we can apply the results of the previous sections concerning stationary solutions. The new stationary solutions can be obtained for example by setting $\bar{\xi}=\left(0,0, \bar{\xi}_{3}, 0,0,0,0, \bar{\xi}_{8}\right)$. Namely, we find $\bar{\xi}_{1,2}=\left(0,0, \pm \frac{\sqrt{3}}{2}, 0,0,0,0, \frac{1}{2}\right)$ and $\overline{\boldsymbol{\xi}}_{3}=(0,0,0,0,0,0,0,-1)$. This solution represents the pure states. It follows from numerical calculations that the solution $\overline{\boldsymbol{\xi}}_{1}$ is asymptotically stable and $\overline{\boldsymbol{\xi}}_{2}$ and $\bar{\xi}_{3}$ are unstable.

\subsection{Rational solution}

We finally discuss the case when the series representing the exponential from (1.1) such that $\mathrm{e}^{-i t(H+i G)}=\mathrm{e}^{-\mathrm{i} t(\boldsymbol{a}+i \boldsymbol{b}) \cdot \lambda}$ truncates and becomes a polynomial, so the solution to (2.9) is rational. This is the only simple example involving nonvanishing both $\boldsymbol{a}$ and $\boldsymbol{b}$. We recall that in the case of the qubit the rational solution was intermediate between the periodic and hyperbolic motion. Taking into account (A.31), we find that whenever $\boldsymbol{a}^{2}=\boldsymbol{b}^{2}, \boldsymbol{a} \cdot \boldsymbol{b}=0$, and $\boldsymbol{a} * \boldsymbol{a}=\boldsymbol{b} * \boldsymbol{b}, \boldsymbol{a} * \boldsymbol{b}=0$, then

$$
\mathrm{e}^{-i t(\boldsymbol{a}+i \boldsymbol{b}) \cdot \lambda}=1-\mathrm{i} t(\boldsymbol{a}+i \boldsymbol{b}) \cdot \boldsymbol{\lambda} .
$$

We remark that the conditions satisfied by $\boldsymbol{a}$ and $\boldsymbol{b}$ in the case of the rational solution are the most natural generalizations of those taking place for the qubit such that $\boldsymbol{a}^{2}=\boldsymbol{b}^{2}$ and $\boldsymbol{a} \cdot \boldsymbol{b}=0$. It should also be noted that relations satisfied by $\boldsymbol{a}$ and $\boldsymbol{b}$ imply $\boldsymbol{a} \cdot(\boldsymbol{a} * \boldsymbol{a})=0$ and $\boldsymbol{b} \cdot(\boldsymbol{b} * \boldsymbol{b})=0$ following directly from (A.27). Now, making use of (4.21) we get after some calculation the following solution to (2.9) expressed by (4.2) with

$$
\begin{aligned}
& \boldsymbol{\eta}(t)=\boldsymbol{\xi}_{0}+\frac{2}{\sqrt{3}}\left(\boldsymbol{b}+\boldsymbol{b} * \boldsymbol{\xi}_{0}+\boldsymbol{a} \wedge \boldsymbol{\xi}_{0}\right) t+\left\{\frac{2}{3} \boldsymbol{a} * \boldsymbol{a}+\frac{2}{3} \boldsymbol{a} \wedge \boldsymbol{b}-\frac{2}{3} \boldsymbol{a}^{2} \boldsymbol{\xi}_{0}\right. \\
& \left.-\frac{4}{3}(\boldsymbol{a} * \boldsymbol{a}) * \boldsymbol{\xi}_{0}+2\left(\boldsymbol{a} \cdot \boldsymbol{\xi}_{0}\right) \boldsymbol{a}+2\left(\boldsymbol{b} \cdot \boldsymbol{\xi}_{0}\right) \boldsymbol{b}-2\left[\boldsymbol{a} *\left(\boldsymbol{b} \wedge \boldsymbol{\xi}_{0}\right)-\boldsymbol{a} \wedge\left(\boldsymbol{b} * \boldsymbol{\xi}_{0}\right)\right]\right\} t^{2}, \\
& \varphi(t)=1+\frac{4 \sqrt{3}}{3} \boldsymbol{b} \cdot \boldsymbol{\xi}_{0} t+\frac{4}{3}\left[\boldsymbol{a}^{2}+(\boldsymbol{a} * \boldsymbol{a}) \cdot \boldsymbol{\xi}_{0}-(\boldsymbol{a} \wedge \boldsymbol{b}) \cdot \boldsymbol{\xi}_{0}\right] t^{2}
\end{aligned}
$$


On putting $\xi_{0}=0$ and taking the limit $\lim _{t \rightarrow \infty} \frac{\eta(t)}{\varphi(t)}$, we arrive at the asymptotically stable stationary solution of the form

$$
\bar{\xi}=\frac{1}{2} \frac{a * a}{a^{2}}+\frac{1}{2} \frac{a \wedge b}{a^{2}}
$$

This stationary solution satisfies $\xi^{2}=\frac{1}{2}$ and $3 \xi^{2}-2 \bar{\xi} \cdot(\bar{\xi} * \bar{\xi})=1$, so it represents a mixed state on the boundary of the space of states.

\subsection{Limit cycle, spiral trajectories and remaining solutions for $a \neq 0$ and $b \neq 0$}

An interesting nontrivial property of the nonlinear system (2.9) absent in the case of the qubit is the existence of the limit cycles. More precisely, it follows from the numerical calculations that whenever $\boldsymbol{a}^{2}>\boldsymbol{b}^{2}, \boldsymbol{a} \cdot \boldsymbol{b}=0, \boldsymbol{a} \cdot(\boldsymbol{a} * \boldsymbol{a})=0, \boldsymbol{a} \cdot(\boldsymbol{b} * \boldsymbol{b})=0$, and $\boldsymbol{b} \cdot(\boldsymbol{a} * \boldsymbol{a})>0$, the trajectories go to the periodic solution represented in the projections onto the planes $\left(\xi_{i}, \xi_{j}\right)$ or $\left(\xi_{i}, \xi_{j}, \xi_{k}\right), i, j, k=1,2, \ldots, 8$ by ellipses. We point out that the first two conditions $\boldsymbol{a}^{2}>\boldsymbol{b}^{2}$ and $\boldsymbol{a} \cdot \boldsymbol{b}=0$ refer to periodic solutions in the case of the nonlinear evolution of the qubit [1]. The limit cycle of the system (2.9) is illustrated in Fig. 5. It should be noted that the parameters of the limit cycles depend on initial data. As with the nonlinear evolution of the qubit, there exist for $\boldsymbol{a} \cdot \boldsymbol{b} \neq 0$ the spiral trajectories going to equilibrium points (stationary solutions) corresponding to the pure states that are combinations of peri-

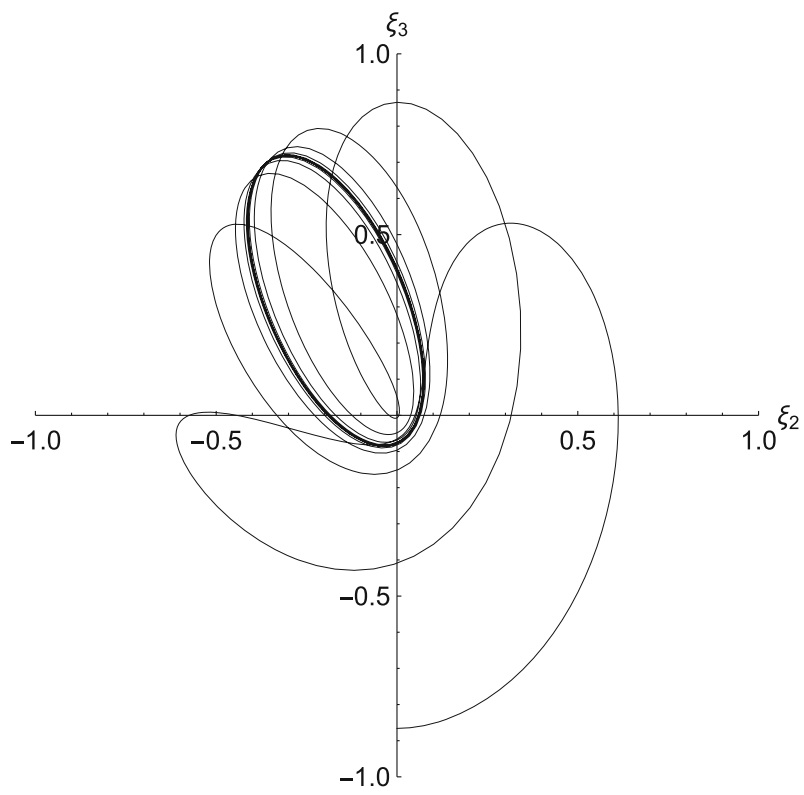

Fig. 5 The limit cycle of the system (2.9), where $\boldsymbol{a}=(1,1,0,2,-2,1,0,0), \boldsymbol{b}=\left(0,0, \frac{\sqrt{3}}{2}, 0,0,0,0, \frac{1}{2}\right)$ and $\xi_{0}=\left(0,0,-\frac{\sqrt{3}}{2}, 0,0,0,0, \frac{1}{2}\right)$ 
odic and hyperbolic motion. An example is of the form $\boldsymbol{a}=(1,1,0,2,-2,1,0,1)$, $\boldsymbol{b}=\left(0,0, \frac{\sqrt{3}}{2}, 0,0,0,0,-\frac{1}{2}\right)$ and $\boldsymbol{\xi}_{0}=(0.1,0.1,0.1,0.1,0.1,0.1,0.1,0.1)$. The explicit solution for $\boldsymbol{a} \cdot \boldsymbol{b} \neq 0$ can be easily obtained by setting $\boldsymbol{a}=\mu \boldsymbol{b}$ and using solutions obtained earlier. For instance, one can demand that $\boldsymbol{b} \cdot(\boldsymbol{b} * \boldsymbol{b})=0$ and use solutions (3.28) and (4.11). In opposition to the case of the qubit, we have also the spiral solutions corresponding to $\boldsymbol{a} \cdot \boldsymbol{b}=0$. The conditions for parameters can be then obtained from that holding in the case of the limit cycle by omitting some of requirements. An example is $\boldsymbol{a}=(0,1,0,2,-2,1,0,0)$ and $\boldsymbol{b}=\left(0,0, \frac{\sqrt{3}}{2}, 0,0,0,0,-\frac{1}{2}\right)$ satisfying $\boldsymbol{a} \cdot(\boldsymbol{a} * \boldsymbol{a}) \neq 0$ as well as $\boldsymbol{a}=(1,1,0,2,-2,1,0,0)$ and $\boldsymbol{b}=$ $\left(0,0, \frac{1}{2}(1-\sqrt{3}), 0,0,0,0, \frac{1}{2}(1-\sqrt{3})\right.$ fulfilling $\boldsymbol{a} \cdot(\boldsymbol{b} * \boldsymbol{b}) \neq 0$ and $\boldsymbol{b} \cdot(\boldsymbol{a} * \boldsymbol{a})<0$. In both examples $\xi_{0}=(0.1,0.1,0.1,0.1,0.1,0.1,0.1,0.1)$. An interesting new type of solutions that occur in the discussed case of the qutrit is dumped quasiperiodic oscillations such that the volume of the phase space occupied by the trajectory shrinks to zero and solution goes to the equilibrium point representing a pure state. Such oscillations that can be regarded as the quasiperiodic counterpart of the spiral motion are depicted in Fig. 6. We finally point out that the Gell-Mann matrices $\lambda_{1}, \lambda_{2}$ and $\lambda_{3}$ are generators of the $S U(2)$ group, so we can recover all solutions obtained in the case of the qubit [1] by putting $\boldsymbol{a}=\left(a_{1}, a_{2}, a_{3}, 0,0,0,0,0\right), \boldsymbol{b}=\left(b_{1}, b_{2}, b_{3}, 0,0,0,0,0\right)$ and $\xi_{0}=\left(\xi_{01}, \xi_{02}, \xi_{03}, 0,0,0,0,0\right)$.

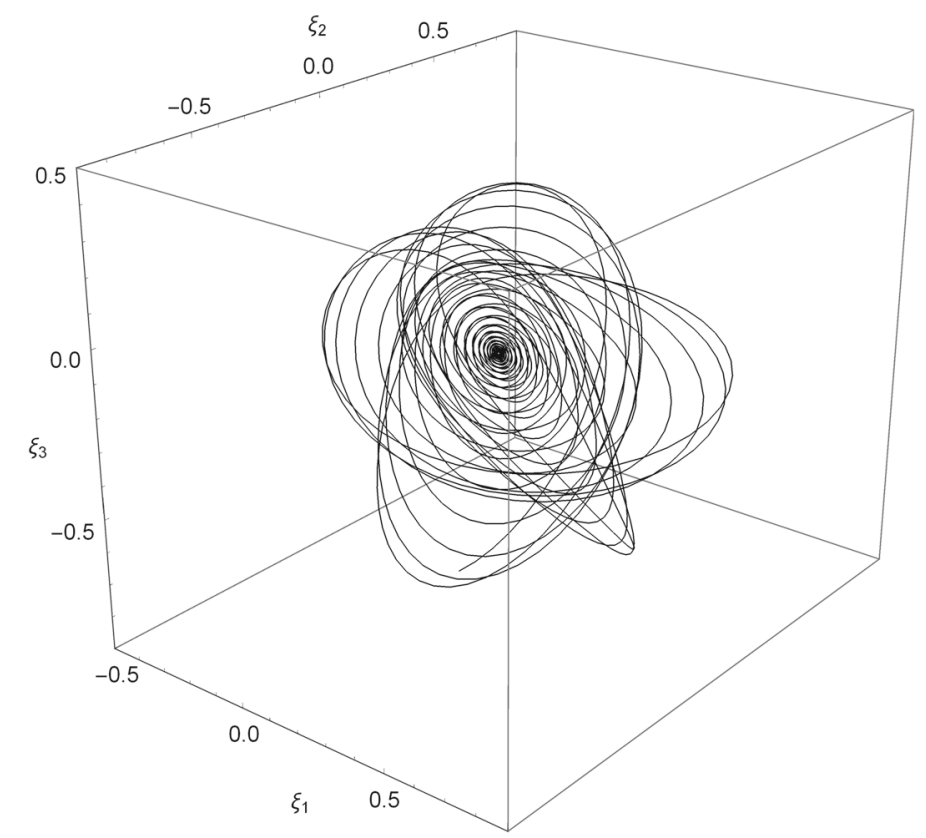

Fig. 6 Trajectory of the system (2.9) with $\boldsymbol{a}=(1,0,-1,0,2,-1,1,-1), \quad \boldsymbol{b}=$ $(0.1,0.1,0.1,0.1,0.1,0.1,0.1,0.1)$ and $\xi_{0}=\left(0,0, \frac{\sqrt{3}}{2}, 0,0,0,0, \frac{1}{2}\right) \quad$ (pure state), of the dumped quasiperiodic motion, going to the stationary (equilibrium) solution $\bar{\xi}=$ $(0.284966,-0.168841,-0.042086,-0.035279,0.556160,-0.356250,0.522711,-0.421682)$ (pure state) 


\section{Dynamics of entropy for qutrit states}

Our purpose now is to study the temporal development of entropy under the evolution of the qutrit state given by (1.1), (2.7) and (2.8). Solving the characteristic equation $\operatorname{det}[\rho(t)-v I]=0$, where $\rho(t)$ is given by (2.7), that is cubic in $v$, we get the following trigonometric solution:

$$
v_{1}=\frac{1}{3}+\frac{2}{3}|\xi(t)| \cos \frac{\alpha}{3}, \quad v_{2,3}=\frac{1}{3}-\frac{2}{3}|\xi(t)| \cos \left(\frac{\alpha}{3} \pm \frac{\pi}{3}\right)
$$

where

$$
\cos \alpha=\frac{\xi(t) \cdot[\xi(t) * \xi(t)]}{|\xi(t)|^{3}}
$$

Hence, we obtain the following expression for the entropy of the qutrit states:

$$
\begin{aligned}
S(t)= & -\operatorname{Tr}\left[\rho(t) \log _{3} \rho(t)\right] \\
= & -\frac{1}{3}\left(1+2|\xi(t)| \cos \frac{\alpha}{3}\right) \log _{3} \frac{1}{3}\left(1+2|\xi(t)| \cos \frac{\alpha}{3}\right) \\
& -\frac{1}{3}\left[1-2|\xi(t)| \cos \left(\frac{\alpha}{3}+\frac{\pi}{3}\right)\right] \log _{3} \frac{1}{3}\left[1-2|\xi(t)| \cos \left(\frac{\alpha}{3}+\frac{\pi}{3}\right)\right] \\
& -\frac{1}{3}\left[1-2|\xi(t)| \cos \left(\frac{\alpha}{3}-\frac{\pi}{3}\right)\right] \log _{3} \frac{1}{3}\left[1-2|\xi(t)| \cos \left(\frac{\alpha}{3}-\frac{\pi}{3}\right)\right] .
\end{aligned}
$$

Notice that the maximum value 1 of the entropy $S(t)$ corresponds to $|\xi(t)|=0$, i.e., maximally mixed state, and its minimum value 0 is reached at $|\boldsymbol{\xi}(t)|=1$, $\xi(t) * \xi(t)=\xi(t)$ referring to the pure state (see (2.4)). The entropy is periodic function of time for periodic solutions to (2.9) corresponding to mixed states and as it

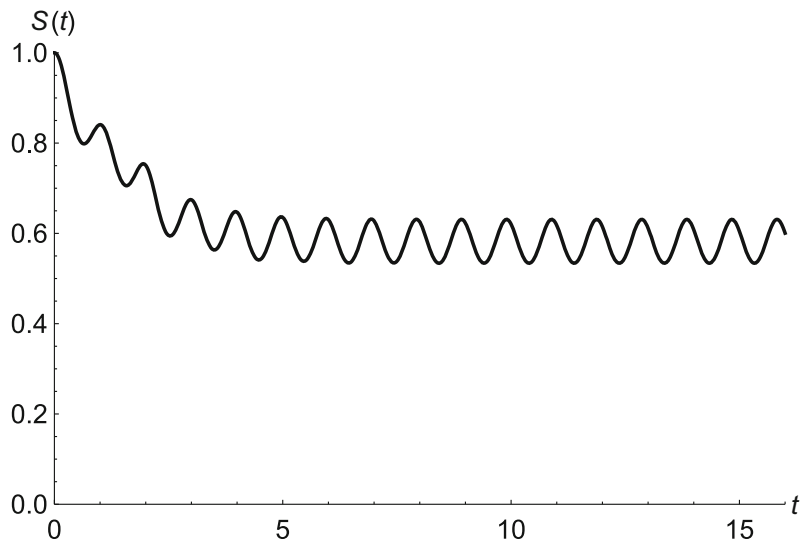

Fig. 7 Auto-oscillations of the entropy of the qutrit given by (5.3) connected with the existence of the limit cycle of the system (2.9), where the parameters are $\boldsymbol{a}=(1,1,0,2,-2,1,0,0), \boldsymbol{b}=$ $\left(0,0, \frac{\sqrt{3}}{2}, 0,0,0,0, \frac{1}{2}\right)$, and $\xi_{0}=(0,0,0,0,0,0,0,0)$ 
follows from numerical calculations, decreasing function of time involving possibility of dumped oscillations, for solutions going to equilibrium points on the boundary of the space of states except of those with the initial data corresponding to pure states. Further, the entropy is constant for stationary solutions and trajectories on the boundary $\partial \Omega_{p}$. The novel behavior of the entropy that is absent in the evolution of qubit states includes the case of the auto-oscillations connected with the existence of the limit cycles illustrated in Fig. 7. Another example is the horizontal asymptotes corresponding to the asymptotically stable stationary solutions connected with the mixed states on the boundary of the state space.

\section{Conclusions}

In this work, we study the nonlinear generalization of the von Neumann equation. An advantage of such nonlinear generalization in comparison with alternative approaches is that it preserves the convexity of the space of quantum states. This means that the introduced nonlinearity does not violate the probabilistic structure of quantum mechanics. Another asset of the approach taken up in this work usually absent in nonlinear generalizations of quantum mechanics is that it does not enable superluminal communication. We point out that the nonlinear generalization of the von Neumann equation describes open quantum systems. This fact was recognized in the special case of the qubit in Ref. [11] based on the applications of non-Hermitian Hamiltonians such as $H+i G$ given by (1.10) in the theory of open quantum systems. The authors presented therein as an illustrative example the observations of the classical paper of Feshbach $[18,19]$ who utilized for the first time non-Hermitian Hamiltonians for description of coupling between a discrete level and a continuum of states of a given quantum system. Such approach is nowadays employed in physics of open quantum systems as for instance phase transitions. Another example is non-Hermiticity appearing in nononequilibrium open systems with gain and/or loss [20-24]. Even more convincing evidence of the openness of the quantum system described by the nonlinear von Neumann equation (1.2) is the global asymptotic stability of stationary solutions to the nonlinear system (1.12) mentioned in Introduction, corresponding to evolution of the qubit from mixed states to the pure ones. In other words, in the discussed case every mixed state from the interior of the Bloch sphere goes to the pure state represented by the point on the sphere. Of course, as depicted in Ref. [1] in Fig. 4, entropy is then decreasing function of time. Clearly, such situation cannot occur in the case of an isolated system.

As a concrete realization of the nonlinear von Neumann equation, we have chosen the nontrivial case of the qutrit. As one would expect, this three-level system exhibits richer dynamics than does the qubit analyzed in the recent work [1]. In particular, the classical Riccati system (2.9) corresponding to the qutrit has quasiperiodic solutions and limit cycles that are absent in the case of the qubit. Interestingly, the quasiperiodic trajectories in the investigated three-level quantum system such as that illustrated in Fig. 3 have counterpart in classical orbital mechanics called Lissajous orbits that an object can follow around the Lagrangian point of a three-body system. The existence of the limit cycles of the system (2.9) and consequently self-oscillations of the qutrit 
is remarkable. The author does not know any other example of the limit cycle in a simple quantum system like that described in this work. For instance, one can find in the literature limit cycles in renormalization group behavior of quantum Hamiltonians [25]. Self-oscillations as dynamical peculiarity of open systems are well known in classical physics. For example, we can read in Ref. [26] that "Self-oscillation system is an open system because emergence and maintenance energy is given by an external source" or in Ref. [27] that "Self-oscillatory systems have some properties that differ from those of harmonic oscillators. First, they exhibit undamped oscillations by taking and dissipating energy from various sources; thus, the systems have typical characteristics of nonequilibrium open systems." Referring to the harmonic oscillator mentioned in the second quotation, we point out that the closed, periodic trajectory depicted in Fig. 5 is reached only asymptotically. An example of connection between self-oscillations of energy and entropy of a mechanical system was provided in Ref. [28]. An important example of self-oscillations in open systems far from equilibrium is the temporal celebrated dissipative structures in the form of sustained oscillations, illustrated by biological rhythms [29] playing the fundamental role in the biological self-organization. Bearing in mind the existence of the limit cycle in the case of the qutrit and its lack for the qubit's time evolution, it is worthwhile to note that occurrence of dissipative structure in the case of chemical reactions takes place for three-particle reactions [30] that are less probable than two-particle ones. Referring back to the ringing structure in the entropy in Fig. 7, we point out an intriguing interpretation of such entropy oscillations [31] as a process of self-organization and process of disorganization of an open system arising after reaching some critical levels of organization. It is further suggested that these oscillations can model action of mankind such as constructive one like: "building houses and factories, partition off rivers by dams and etc." and destructive: such as "disintegration of ecosystems, destructive fluctuations of the climate" and so on. As an advantage of this approach, the author of Ref. [31] indicates that "The understanding of the reasons of the given tendency not only enables to foresee future hardships, but also prompts a way to avoid them."

The fundamental character of the notion of the qutrit in quantum information theory suggests that the observations of this work concerning the nonlinear evolution of the qutrit would be of importance in quantum information processing. We also remark that some of the identities presented in "Appendix" concerning star and wedge products related to the structure constants of the $s u(3)$ algebra are most probably new. It seems that they would be a useful tool in the study of the qutrit and other problems connected with the $S U(3)$ symmetry.

Acknowledgements I would like to thank Jakub Rembieliński for helpful comments.

Open Access This article is licensed under a Creative Commons Attribution 4.0 International License, which permits use, sharing, adaptation, distribution and reproduction in any medium or format, as long as you give appropriate credit to the original author(s) and the source, provide a link to the Creative Commons licence, and indicate if changes were made. The images or other third party material in this article are included in the article's Creative Commons licence, unless indicated otherwise in a credit line to the material. If material is not included in the article's Creative Commons licence and your intended use is not permitted by statutory regulation or exceeds the permitted use, you will need to obtain permission directly from the copyright holder. To view a copy of this licence, visit http://creativecommons.org/licenses/by/4.0/. 


\section{Appendix}

We begin by collecting the basic properties of the Gell-Mann matrices:

$$
\begin{aligned}
\lambda_{1}=\left(\begin{array}{lll}
0 & 1 & 0 \\
1 & 0 & 0 \\
0 & 0 & 0
\end{array}\right), & \lambda_{2}=\left(\begin{array}{ccc}
0 & -i & 0 \\
i & 0 & 0 \\
0 & 0 & 0
\end{array}\right), & \lambda_{3}=\left(\begin{array}{ccc}
1 & 0 & 0 \\
0 & -1 & 0 \\
0 & 0 & 0
\end{array}\right), \\
\lambda_{4}=\left(\begin{array}{lll}
0 & 0 & 1 \\
0 & 0 & 0 \\
1 & 0 & 0
\end{array}\right), & \lambda_{5}=\left(\begin{array}{ccc}
0 & 0 & -i \\
0 & 0 & 0 \\
i & 0 & 0
\end{array}\right), & \\
\lambda_{6}=\left(\begin{array}{lll}
0 & 0 & 0 \\
0 & 0 & 1 \\
0 & 1 & 0
\end{array}\right), & \lambda_{7}=\left(\begin{array}{ccc}
0 & 0 & 0 \\
0 & 0 & -i \\
0 & i & 0
\end{array}\right), & \lambda_{8}=\frac{1}{\sqrt{3}}\left(\begin{array}{ccc}
1 & 0 & 0 \\
0 & 1 & 0 \\
0 & 0 & -2
\end{array}\right) .
\end{aligned}
$$

These matrices are Hermitian and traceless. They obey the following commutation relations:

$$
\begin{aligned}
{\left[\lambda_{j}, \lambda_{k}\right] } & =2 i f_{j k l} \lambda_{l}, \\
\left\{\lambda_{j}, \lambda_{k}\right\} & =\frac{4}{3} \delta_{j k}+2 d_{j k l} \lambda_{l},
\end{aligned}
$$

where the coefficients (structure constants of the $s u(3)$ algebra) $f_{j k l}$ are completely antisymmetric and $d_{j k l}$ are completely symmetric. The independent, nonvanishing components of $f_{j k l}$ and $d_{j k l}$ are given by

$$
\begin{aligned}
& f_{123}=1, \quad f_{458}=f_{678}=\frac{\sqrt{3}}{2}, \\
& f_{147}=f_{246}=f_{257}=f_{345}=f_{516}=f_{637}=\frac{1}{2},
\end{aligned}
$$

and

$$
\begin{aligned}
& d_{118}=d_{228}=d_{338}=-d_{888}=\frac{1}{\sqrt{3}}, \\
& d_{448}=d_{558}=d_{668}=d_{778}=-\frac{1}{2 \sqrt{3}}, \\
& d_{146}=d_{157}=-d_{247}=d_{256}=d_{344}=d_{355}=-d_{366}=-d_{377}=\frac{1}{2} .
\end{aligned}
$$

We define with the help of the coefficients $f_{i j k}$ and $d_{i j k}$, respectively, the antisymmetric wedge product of vectors $\boldsymbol{a}, \boldsymbol{b} \in \mathbb{R}^{8}$ :

$$
(\boldsymbol{a} \wedge \boldsymbol{b})_{i}=\sqrt{3} f_{i j k} a_{j} b_{k}
$$


and the symmetric star product

$$
(\boldsymbol{a} * \boldsymbol{b})_{i}=\sqrt{3} d_{i j k} a_{j} b_{k}
$$

Using (A.4) and (A.6), we find that the coordinates of the vector $\boldsymbol{a} \wedge \boldsymbol{b}$ are

$$
\begin{aligned}
(\boldsymbol{a} \wedge \boldsymbol{b})_{1}= & \sqrt{3}\left[a_{2} b_{3}-a_{3} b_{2}+\frac{1}{2}\left(a_{4} b_{7}-a_{7} b_{4}\right)+\frac{1}{2}\left(a_{6} b_{5}-a_{5} b_{6}\right)\right], \\
(\boldsymbol{a} \wedge \boldsymbol{b})_{2}= & \left.\sqrt{3}\left[\frac{1}{2}\left(a_{4} b_{6}-a_{6} b_{4}\right)+\frac{1}{2}\left(a_{5} b_{7}-a_{7} b_{5}\right)+a_{3} b_{1}-a_{1} b_{3}\right)\right], \\
(\boldsymbol{a} \wedge \boldsymbol{b})_{3}= & \left.\sqrt{3}\left[\frac{1}{2}\left(a_{4} b_{5}-a_{5} b_{4}\right)+\frac{1}{2}\left(a_{7} b_{6}-a_{6} b_{7}\right)+a_{1} b_{2}-a_{2} b_{1}\right)\right], \\
(\boldsymbol{a} \wedge \boldsymbol{b})_{4}= & \sqrt{3}\left[\frac{\sqrt{3}}{2}\left(a_{5} b_{8}-a_{8} b_{5}\right)+\frac{1}{2}\left(a_{7} b_{1}-a_{1} b_{7}\right)\right. \\
& \left.+\frac{1}{2}\left(a_{6} b_{2}-a_{2} b_{6}\right)+\frac{1}{2}\left(a_{5} b_{3}-a_{3} b_{5}\right)\right], \\
(\boldsymbol{a} \wedge \boldsymbol{b})_{5}= & \sqrt{3}\left[\frac{1}{2}\left(a_{1} b_{6}-a_{6} b_{1}\right)+\frac{1}{2}\left(a_{3} b_{4}-a_{4} b_{3}\right)\right. \\
& \left.+\frac{1}{2}\left(a_{7} b_{2}-a_{2} b_{7}\right)+\frac{\sqrt{3}}{2}\left(a_{8} b_{4}-a_{4} b_{8}\right)\right], \\
(\boldsymbol{a} \wedge \boldsymbol{b})_{6}= & \sqrt{3}\left[p g \frac{1}{2}\left(a_{3} b_{7}-a_{7} b_{3}\right)+\frac{1}{2}\left(a_{5} b_{1}-a_{1} b_{5}\right)\right. \\
& \left.+\frac{1}{2}\left(a_{2} b_{4}-a_{4} b_{2}\right)+\frac{\sqrt{3}}{2}\left(a_{7} b_{8}-a_{8} b_{7}\right)\right], \\
(\boldsymbol{a} \wedge \boldsymbol{b})_{7}= & \sqrt{3}\left[\frac{\sqrt{3}}{2}\left(a_{8} b_{6}-a_{6} b_{8}\right)+\frac{1}{2}\left(a_{1} b_{4}-a_{4} b_{1}\right)\right. \\
& \left.+\frac{1}{2}\left(a_{2} b_{5}-a_{5} b_{2}\right)+\frac{1}{2}\left(a_{6} b_{3}-a_{3} b_{6}\right)\right], \\
(\boldsymbol{a} \wedge \boldsymbol{b})_{8}= & \sqrt{3}\left[\frac{\sqrt{3}}{2}\left(a_{4} b_{5}-a_{5} b_{4}\right)+\frac{\sqrt{3}}{2}\left(a_{6} b_{7}-a_{7} b_{6}\right)\right] .
\end{aligned}
$$

Furthermore, making use of (A.5) and (A.7) we get the coordinates of the vector $\boldsymbol{a} * \boldsymbol{b}$

$$
\begin{aligned}
(\boldsymbol{a} * \boldsymbol{b})_{1}= & \sqrt{3}\left[\frac{1}{\sqrt{3}}\left(a_{1} b_{8}+a_{8} b_{1}\right)+\frac{1}{2}\left(a_{4} b_{6}+a_{6} b_{4}\right)+\frac{1}{2}\left(a_{5} b_{7}+a_{7} b_{5}\right)\right], \\
(\boldsymbol{a} * \boldsymbol{b})_{2}= & \sqrt{3}\left[\frac{1}{\sqrt{3}}\left(a_{2} b_{8}+a_{8} b_{2}\right)-\frac{1}{2}\left(a_{4} b_{7}+a_{7} b_{4}\right)+\frac{1}{2}\left(a_{5} b_{6}+a_{6} b_{5}\right)\right], \\
(\boldsymbol{a} * \boldsymbol{b})_{3}= & \sqrt{3}\left[\frac{1}{\sqrt{3}}\left(a_{3} b_{8}+a_{8} b_{3}\right)+\frac{1}{2}\left(a_{4} b_{4}+a_{5} b_{5}-a_{6} b_{6}-a_{7} b_{7}\right)\right], \\
(\boldsymbol{a} * \boldsymbol{b})_{4}= & \sqrt{3}\left[-\frac{1}{2 \sqrt{3}}\left(a_{4} b_{8}+a_{8} b_{4}\right)+\frac{1}{2}\left(a_{1} b_{6}+a_{6} b_{1}\right)\right. \\
& \left.-\frac{1}{2}\left(a_{7} b_{2}+a_{2} b_{7}\right)+\frac{1}{2}\left(a_{3} b_{4}+a_{4} b_{3}\right)\right],
\end{aligned}
$$




$$
\begin{aligned}
(\boldsymbol{a} * \boldsymbol{b})_{5}= & \sqrt{3}\left[-\frac{1}{2 \sqrt{3}}\left(a_{5} b_{8}+a_{8} b_{5}\right)+\frac{1}{2}\left(a_{1} b_{7}+a_{7} b_{1}\right)\right. \\
& \left.+\frac{1}{2}\left(a_{2} b_{6}+a_{6} b_{2}\right)+\frac{1}{2}\left(a_{3} b_{5}+a_{5} b_{3}\right)\right] \\
(\boldsymbol{a} * \boldsymbol{b})_{6}= & \sqrt{3}\left[-\frac{1}{2 \sqrt{3}}\left(a_{6} b_{8}+a_{8} b_{6}\right)+\frac{1}{2}\left(a_{1} b_{4}+a_{4} b_{1}\right)\right. \\
& \left.+\frac{1}{2}\left(a_{2} b_{5}+a_{5} b_{2}\right)-\frac{1}{2}\left(a_{3} b_{6}+a_{6} b_{3}\right)\right] \\
(\boldsymbol{a} * \boldsymbol{b})_{7}= & \sqrt{3}\left[-\frac{1}{2 \sqrt{3}}\left(a_{7} b_{8}+a_{8} b_{7}\right)+\frac{1}{2}\left(a_{1} b_{5}+a_{5} b_{1}\right)\right. \\
& \left.-\frac{1}{2}\left(a_{2} b_{4}+a_{4} b_{2}\right)-\frac{1}{2}\left(a_{3} b_{7}+a_{7} b_{3}\right)\right] \\
(\boldsymbol{a} * \boldsymbol{b})_{8}= & \sqrt{3}\left[\frac{1}{\sqrt{3}}\left(a_{1} b_{1}+a_{2} b_{2}+a_{3} b_{3}-a_{8} b_{8}\right)\right. \\
& \left.-\frac{1}{2 \sqrt{3}}\left(a_{4} b_{4}+a_{5} b_{5}+a_{6} b_{6}+a_{7} b_{7}\right)\right]
\end{aligned}
$$

In the particular case of $\boldsymbol{a}=\left(0,0, a_{3}, 0,0,0,0, a_{8}\right)$ and $\boldsymbol{b}=\left(0,0, b_{3}, 0,0,0,0, b_{8}\right)$, we have

$$
\begin{aligned}
& (\boldsymbol{a} * \boldsymbol{b})_{3}=a_{3} b_{8}+a_{8} b_{3}, \\
& (\boldsymbol{a} * \boldsymbol{b})_{8}=a_{3} b_{3}-a_{8} b_{8},
\end{aligned}
$$

where the remaining coordinates of $\boldsymbol{a} * \boldsymbol{b}$ vanish. We now write down some identities satisfied by the wedge and the star products. We begin with the relation implied by the Jacobi identity satisfied by the coefficients $f_{i j k}$ such that

$$
a \wedge(b \wedge c)+b \wedge(c \wedge a)+c \wedge(a \wedge b)=0 .
$$

Using the identity [14]

$$
f_{i j k} f_{k l m}=\frac{2}{3}\left(\delta_{i l} \delta_{j m}-\delta_{i m} \delta_{j l}\right)+d_{i l k} d_{j m k}-d_{j l k} d_{i m k}
$$

we get

$$
\boldsymbol{a} \wedge(\boldsymbol{b} \wedge \boldsymbol{c})=2[\boldsymbol{b}(\boldsymbol{a} \cdot \boldsymbol{c})-\boldsymbol{c}(\boldsymbol{a} \cdot \boldsymbol{b})]+\boldsymbol{b} *(\boldsymbol{a} * \boldsymbol{c})-\boldsymbol{c} *(\boldsymbol{a} * \boldsymbol{b}),
$$

and

$$
(\boldsymbol{a} \wedge \boldsymbol{b})^{2}=\frac{2}{3}\left[\boldsymbol{a}^{2} \boldsymbol{b}^{2}-(\boldsymbol{a} \cdot \boldsymbol{b})^{2}\right]+\frac{1}{3}\left[(\boldsymbol{a} * \boldsymbol{a}) \cdot(\boldsymbol{b} * \boldsymbol{b})-(\boldsymbol{a} * \boldsymbol{b})^{2}\right]
$$

The next identity [32]

$$
f_{i j k} d_{k l m}+f_{i l k} d_{j m k}+f_{i m k} d_{j l k}=0
$$


implies

$$
a \wedge(b * c)+b \wedge(c * a)+c \wedge(a * b)=0 .
$$

Now, taking into account the identity [14]

$$
f_{i j k} d_{k l m}+f_{l j k} d_{i m k}+f_{m j k} d_{i l k}=0,
$$

we find

$$
a \wedge(b * c)+c *(b \wedge a)+b *(c \wedge a)=0 .
$$

An immediate consequence of (A.16) or (A.18) is

$$
a \wedge(a * a)=0 .
$$

Taking into account (A.19), one can show [16] that

$$
\boldsymbol{a} \wedge \boldsymbol{b}=0 \Longleftrightarrow \boldsymbol{b}=\mu \boldsymbol{a}+\nu \boldsymbol{a} * \boldsymbol{a},
$$

where $\mu$ and $v$ are constant. Further, (A.16) and (A.18) taken together yield

$$
b \wedge(a * c)+c \wedge(a * b)=c *(b \wedge a)+b *(c \wedge a) .
$$

Finally, the identity [32]

$$
d_{i j k} d_{k l m}+d_{i l k} d_{k j m}+d_{i m k} d_{k j l}=\frac{1}{3}\left(\delta_{i j} \delta_{l m}+\delta_{i l} \delta_{j m}+\delta_{i m} \delta_{j l}\right)
$$

leads to

$$
\boldsymbol{a} *(\boldsymbol{b} * \boldsymbol{c})+\boldsymbol{b} *(\boldsymbol{c} * \boldsymbol{a})+\boldsymbol{c} *(\boldsymbol{a} * \boldsymbol{b})=\boldsymbol{a}(\boldsymbol{b} \cdot \boldsymbol{c})+\boldsymbol{b}(\boldsymbol{c} \cdot \boldsymbol{a})+\boldsymbol{c}(\boldsymbol{a} \cdot \boldsymbol{b}) .
$$

In the special case $\boldsymbol{a}=\boldsymbol{b}=\boldsymbol{c}$, we get from (A.23) the useful relation

$$
a *(a * a)=a^{2} a .
$$

On the other hand, using (A.23) and (A.24) we obtain

$$
(\boldsymbol{a} * \boldsymbol{a}) *(\boldsymbol{a} * \boldsymbol{a})=2[\boldsymbol{a} \cdot(\boldsymbol{a} * \boldsymbol{a})] \boldsymbol{a}-\boldsymbol{a}^{2} \boldsymbol{a} * \boldsymbol{a} .
$$

We supplement the identities concerning wedge and star product with the following quite obvious relations involving scalar product:

$$
\begin{gathered}
a \cdot(b \wedge c)=b \cdot(c \wedge a)=c \cdot(a \wedge b), \\
a \cdot(b * c)=b \cdot(c * a)=c \cdot(a * b) .
\end{gathered}
$$


From (A.24) and (A.27), it follows that

$$
(\boldsymbol{a} * \boldsymbol{a})^{2}=|\boldsymbol{a}|^{4}
$$

where $|\boldsymbol{a}|=\sqrt{\boldsymbol{a}^{2}}$ is the Euclidean norm of the vector $\boldsymbol{a}$.

We end this section with algebraic relations for the Gell-Mann matrices. We first write down the formula for the product of these matrices following directly from (A.2) and (A.3):

$$
\lambda_{j} \lambda_{k}=\frac{2}{3} \delta_{j k}+\left(d_{j k l}+\mathrm{i} f_{j k l}\right) \lambda_{l}
$$

Hence, we get

$$
(\boldsymbol{a} \cdot \lambda)(\boldsymbol{b} \cdot \lambda)=\frac{2}{3} \boldsymbol{a} \cdot \boldsymbol{b}+\left[\frac{1}{\sqrt{3}} \boldsymbol{a} * \boldsymbol{b}+\frac{\mathrm{i}}{\sqrt{3}} \boldsymbol{a} \wedge \boldsymbol{b}\right] \cdot \lambda .
$$

As an immediate consequence of the relation (A.30), we have

$$
\begin{aligned}
& (\boldsymbol{a} \cdot \lambda)^{2}=\frac{2}{3} \boldsymbol{a}^{2}+\frac{1}{\sqrt{3}}(\boldsymbol{a} * \boldsymbol{a}) \cdot \lambda, \\
& {[\boldsymbol{a} \cdot \lambda, \boldsymbol{b} \cdot \lambda]=\frac{2 \mathrm{i}}{\sqrt{3}}(\boldsymbol{a} \wedge \boldsymbol{b}) \cdot \lambda,} \\
& \{\boldsymbol{a} \cdot \boldsymbol{\lambda}, \boldsymbol{b} \cdot \lambda\}=\frac{4}{3} \boldsymbol{a} \cdot \boldsymbol{b}+\frac{2}{\sqrt{3}}(\boldsymbol{a} * \boldsymbol{b}) \cdot \lambda .
\end{aligned}
$$

Using (A.13) and (A.30), we obtain

$$
\begin{aligned}
& (\boldsymbol{a} \cdot \boldsymbol{\lambda})(\boldsymbol{b} \cdot \lambda)(\boldsymbol{c} \cdot \lambda)=\frac{2}{3 \sqrt{3}}[\boldsymbol{c} \cdot(\boldsymbol{a} * \boldsymbol{b})+i \boldsymbol{c} \cdot(\boldsymbol{a} \wedge \boldsymbol{b})] \\
& \quad+\left\{-\frac{1}{3}[\boldsymbol{b} *(\boldsymbol{a} * \boldsymbol{c})-\boldsymbol{c} *(\boldsymbol{a} * \boldsymbol{b})-\boldsymbol{a} *(\boldsymbol{b} * \boldsymbol{c})]-\frac{2}{3}[\boldsymbol{b}(\boldsymbol{a} \cdot \boldsymbol{c})-\boldsymbol{c}(\boldsymbol{a} \cdot \boldsymbol{b})-\boldsymbol{a}(\boldsymbol{b} \cdot \boldsymbol{c})]\right. \\
& \left.\quad+\frac{i}{3}[\boldsymbol{c} *(\boldsymbol{a} \wedge \boldsymbol{b})-\boldsymbol{c} \wedge(\boldsymbol{a} * \boldsymbol{b})]\right\} \cdot \lambda .
\end{aligned}
$$

For $\boldsymbol{c}=\boldsymbol{a}$, formula (A.34) reduces to

$$
(\boldsymbol{a} \cdot \boldsymbol{\lambda})(\boldsymbol{b} \cdot \boldsymbol{\lambda})(\boldsymbol{a} \cdot \boldsymbol{\lambda})=\frac{2}{3 \sqrt{3}} \boldsymbol{a} \cdot(\boldsymbol{a} * \boldsymbol{b})+\left[-\frac{2}{3} \boldsymbol{b} *(\boldsymbol{a} * \boldsymbol{a})-\frac{1}{3} \boldsymbol{a}^{2} \boldsymbol{b}+2(\boldsymbol{a} \cdot \boldsymbol{b}) \boldsymbol{a}\right] \cdot \boldsymbol{\lambda}
$$

following directly from (A.18) and (A.23). A direct consequence of (A.24) and (A.35) is the relation

$$
(\boldsymbol{a} \cdot \lambda)^{3}=\frac{2}{3 \sqrt{3}} \boldsymbol{a} \cdot(\boldsymbol{a} * \boldsymbol{a})+\boldsymbol{a}^{2} \boldsymbol{a} \cdot \boldsymbol{\lambda}
$$


We finally write down the trace-orthogonality relations

$$
\operatorname{Tr}\left(\lambda_{j} \lambda_{k}\right)=2 \delta_{j k}
$$

and the invariants of the $S U(3)$ group related to the Casimir operators such that

$$
\begin{aligned}
\frac{1}{2} \operatorname{Tr}(\boldsymbol{a} \cdot \lambda)^{2} & =\boldsymbol{a}^{2}, \\
\frac{\sqrt{3}}{2} \operatorname{Tr}(\boldsymbol{a} \cdot \lambda)^{3} & =\boldsymbol{a} \cdot(\boldsymbol{a} * \boldsymbol{a})
\end{aligned}
$$

implied by (A.31), (A.36) and (A.37). The second invariant (A.39) is related to the determinant of the matrix $\boldsymbol{a} \cdot \boldsymbol{\lambda}$ by

$$
\operatorname{det}(\boldsymbol{a} \cdot \lambda)=\frac{2}{3 \sqrt{3}} \boldsymbol{a} \cdot(\boldsymbol{a} * \boldsymbol{a}) .
$$

This invariant can be written in the coordinate form as

$$
\begin{aligned}
& \boldsymbol{a} \cdot(\boldsymbol{a} * \boldsymbol{a})=3 a_{8}\left(a_{1}^{2}+a_{2}^{2}+a_{3}^{2}\right)-a_{8}^{3}-\frac{3}{2} a_{8}\left(a_{4}^{2}+a_{5}^{2}+a_{6}^{2}+a_{7}^{2}\right) \\
& \quad+\frac{3 \sqrt{3}}{2} a_{3}\left(a_{4}^{2}+a_{5}^{2}-a_{6}^{2}-a_{7}^{2}\right)+3 \sqrt{3}\left[\left(a_{1} a_{6}-a_{2} a_{7}\right) a_{4}+\left(a_{1} a_{7}+a_{2} a_{6}\right) a_{5}\right] .
\end{aligned}
$$

\section{References}

1. Kowalski, K., Rembieliński, J.: Integrable nonlinear evolution of the qubit. Ann. Phys. 411, 167955 (2019)

2. Gisin, N.: Irreversible quantum dynamics and the Hilbert space structure of quantum kinematics. J. Math. Phys. 24(7), 1779 (1983)

3. Turski, Ł.A.: Dissipative quantum mechanics. Metriplectic dynamics in action. In: From Quantum Mechanics to Technology. Lecture Notes in Physics 477, 347 (2007)

4. Grigorenko, A.N.: Measurement description by means of a nonlinear Schrodinger equation. J. Phys. A Math. Gen. 28, 1459 (1995)

5. Kraus, K.: States, Effects, and Operations: Fundamental Notions of Quantum Theory. Springer, Berlin (1983)

6. Grabowski, J., Kuś, M., Marmo, G.: Symmetries, group actions, and entanglement. Open Syst. Inf. Dyn. 13, 343 (2006)

7. Rembieliński, J., Caban, P.: Nonlinear evolution and signaling. Phys. Rev. Res. 2, 012027(R) (2020)

8. Diósi, L.: Nonlinear Schrödinger equation in foundations: summary of 4 catches. J. Phys. Conf. Ser. 701, 012019 (2016)

9. Lamb Jr., W.E.: Theory of an Optical Maser. Phys. Rev. 134, A1429 (1964)

10. Bargmann, V., Michel, L., Telegdi, V.L.: Precession of the polarization of particles moving in a homogeneous electromagnetic field. Phys. Rev. Lett. 2, 435 (1959)

11. Grimaudo, R., de Castro, A.S.M., Kuś, Messina, A.: Exactly solvable time-dependent pseudoHermitian su(1,1) Hamiltonian models. Phys. Rev. A 98, 033835 (2018)

12. Rembieliński, J., Caban, P.: Nonlinear extension of the quantum dynamical semigroup, 2020 (unpublished) 
13. Goyal, S.K., Simon, B.N., Singh, R., Simon, S.: Geometry of the generalized Bloch sphere for qutrits. J. Phys. A Math. Theor. 49, 165203 (2016)

14. Arvind Mallesh, K.S., Mukunda, N.: A generalized Pancharatnam geometric phase formula for threelevel quantum systems. J. Phys. A Math. Gen. 30, 2417 (1997)

15. Korn, G.A., Korn, T.M.: Mathematical Handbook for Scientists and Engineers. Dover, New York (2000)

16. Mallesh, K.S., Mukunda, N.: The algebra and geometry of SU(3) matrices. Pramana 49, 371 (1997)

17. Bölükbal, A., Dereli, T.: On the $S U$ (3) parametrization of qutrits. J. Phys. Conf. Ser. 36, 28 (2006)

18. Feshbach, H.: Unified theory of nuclear reactions. Ann. Phys. 5, 375 (1958)

19. Feshbach, H.: Unified theory of nuclear reactions. Ann. Phys. 19, 287 (1962)

20. Konotop, V.V., Yang, J., Zezyulin, D.A.: Nonlinear waves in PT-symmetric systems. Rev. Mod. Phys. 88, 035002 (2016)

21. Feng, L., El-Ganainy, R., Ge, L.: Non-Hermitian photonics based on parity-time symmetry. Nat. Photon. 11, 752 (2017)

22. El-Ganainy, R., Makris, K.G., Khajavikhan, M., Musslimani, Z.H., Rotter, S., Christodoulides, D.N.: Non-Hermitian physics and PT symmetry. Nat. Phys. 14, 11 (2018)

23. Miri, M.-A., Alú, A.: Exceptional points in optics and photonics. Science 363, 7709 (2019)

24. Özdemir, Ş.K., Rotter, S., Nori, F., Yang, L.: Parity-time symmetry and exceptional points in photonics. Nat. Mater. 18, 783 (2019)

25. Głazek, S.D.: Limit cycles in quantum mechanics. In: Asch, J., Joye, A. (eds.) Mathematical Physics of Quantum Mechanics. Lecture Notes in Physics, vol. 690, p. 65 (2006)

26. Alkhasova, D.A., Sokotushchenko, V.N., Torchinsky, V.M., Zaichenko, V.M.: Peculiarities of excitation of self-oscillations in geological systems. IOP Conf. Ser. Earth Environ. Sci. 249, 012026 (2019)

27. Kinoshita, S.: Pattern Formations and Oscillatory Phenomena. Elsevier, Amsterdam (2013)

28. Petrov, V.V., Ageev, V.M.: Entropy and auto-oscillations in nonlinear systems. Dokl. Akad. Nauk SSSR 199, 1260 (1971)

29. Goldbeter, A.: Dissipative structures in biological systems: bistability, oscillations, spatial patterns and waves. Philos. Trans. R. Soc. A 376, 20170376 (2018)

30. Nicolis, G., Prigogine, I.: Self-organization in Nonequilibrium Systems. Wiley, New York (1977)

31. Shapovalov, V.I.: The criterion of ordering and self-organization of open system. Entropy oscillations in linear and nonlinear processes. Int. J. Appl. Math. Stat. 26, 16-29 (2012). Shapovalov, V.I.: Entropy oscillations, arXiv:0812.4031

32. Haber, H.E.: Useful relations among the generators in the defining and adjoint representations of $S U(N)$ (2017) (unpublished) http://scipp.ucsc.edu/ haber/ph218/sunid17.pdf

Publisher's Note Springer Nature remains neutral with regard to jurisdictional claims in published maps and institutional affiliations. 